\title{
Diagnóstico sobre hábitos de estudio en universitarios de nuevo ingreso como herramienta para identificar oportunidades de mejora
}

\begin{abstract}
Diagnosis of Study Habits in New University Students as a Tool to Identify Opportunities for Improvement
\end{abstract}

Diagnóstico de hábitos de estudo em novos estudantes universitários como ferramenta para identificar oportunidades de melhoria

Juan Carlos Román Fuentes

Universidad Autónoma de Chiapas, México juancrf@gmail.com https://orcid.org/0000-0003-4007-3717

Rafael Timoteo Franco Gurría Universidad Autónoma de Chiapas, México rfrank3@gmail.com https://orcid.org/0000-0001-7330-6113

Rebeca Román Julián Universidad Autónoma de Chiapas, México romanjulianrebeca@gmail.com https://orcid.org/0000-0001-6119-1910 


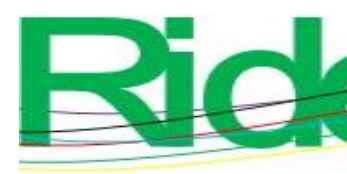

Revista Iberoamericana para la Investigación y el Desarrollo Educativo ISSN 2007 - 7467

\section{Resumen}

Con el objetivo de diagnosticar los hábitos que los universitarios de nuevo ingreso declaran cuando desarrollan actividades relacionadas con el estudio, para identificar áreas de oportunidad que sirvan como referente al emprender procesos de rediseño curricular y de planeación académica, esta aportación indaga sobre las prácticas que los estudiantes suelen realizar de forma cotidiana cuando se trata de estudiar. Esto mediante la aplicación de un test que identifica hábitos que los estudiantes poseen cuando son matriculados en alguna de las cinco licenciaturas (Administración, Contaduría, Gestión Turística, Sistemas Computacionales y Diseño de Software) que ofrece la Facultad de Contaduría y Administración, Campus I (FCA, CI) de la Universidad Autónoma de Chiapas (Unach). El instrumento utilizado se adaptó del planteado por Huidobro, Gutiérrez y Condemarín (2000). Así, al final, constó de 39 reactivos con respuestas dicotómicas (Sí / No), a través de las cuales se midieron ocho dimensiones: Ambiente físico, Estado fisiológico, Distribución de tiempo, Lectura, Técnicas de estudio, Preparación de pruebas, Concentración y Actitud hacia el estudio. Mediante plataforma electrónica, la aplicación fue autoadministrada, y se abarcó a toda la población matriculada en las cinco licenciaturas de cada ciclo escolar participante: un total de 1630 estudiantes. De manera general, los resultados arrojan que en promedio cerca de 8 (7.9) de cada 10 estudiantes tiene dificultades para realizar actividades relacionadas con la lectura y 6 (5.9) de cada 10 declara problemas para distribuir el tiempo. Asimismo, a nivel global, en promedio $23 \%$ de los participantes obtiene ponderaciones aceptables solo en dos de las ocho dimensiones exploradas (aunque por licenciatura este promedio presenta diferencias importantes); $26 \%$ entre tres y cuatro; $30 \%$ entre cinco y seis, y solo $21 \%$ declara siete u ocho dimensiones aceptables. Estos resultados podrán emplearse como referentes para diseñar en el futuro programas sistemáticos y diferenciados de intervención académica que permitan fomentar y mejorar la lectura, las técnicas de distribución de tiempo, la mejora de la concentración y la creación de las condiciones físicas propicias para estudiar, e incluso para diseñar programas de capacitación sistemática del personal docente.

Palabras clave: educación, enseñanza superior, estudiante universitario, proceso de aprendizaje. 


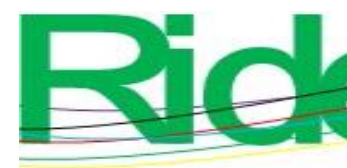

Revista Iberoamericana para la Investigación y el Desarrollo Educativo ISSN $2007-7467$

\section{Abstract}

In order to diagnose the habits that new university students declare when they develop academic activities, to identify opportunity areas that serve as a reference when undertaking processes of curriculum redesign and academic planning, this contribution investigates the practices that students usually perform on a daily basis when it comes to studying. The above through the application of a test that identifies habits that students have when they are enrolled in any of the five degrees (Administration, Accounting, Tourism Management, Computer Systems and Software Development) offered by the Facultad de Contaduría y Administración, Campus I (FCA, CI) of the Universidad Autónoma de Chiapas (Unach). The instrument used was adapted from that proposed by Huidobro, Gutiérrez and Condemarín (2000). Thus, in the end, it consisted of 39 items with dichotomous responses (Yes / No), through which eight dimensions were measured: physical environment, physiological state, time distribution, reading, study techniques, test preparation, concentration and attitude towards study. Through an electronic platform, the application was self-administered, and the entire population enrolled in the five degrees of each participating school year was covered: a total of 1630 students. In general, the results show that on average about 8 (7.9) out of every 10 students have difficulties to carry out activities related to reading, and 6 (5.9) out of 10 declare problems to distribute time. Likewise, globally, on average $23 \%$ of the participating students obtain acceptable weightings only in two of the eight dimensions explored (although by degree, this average presents important differences); $26 \%$ between three and four; $30 \%$ between five and six, and only $21 \%$ declare seven or eight dimensions acceptable. These results can be used as benchmarks to design in the future systematic and differentiated programs of academic intervention that allow the promotion and improvement of reading, the techniques of time distribution, the improvement of concentration and the creation of the physical conditions conducive to studying and even to design systematic training programs for teaching staff.

Keywords: education, higher education, university student, learning process 


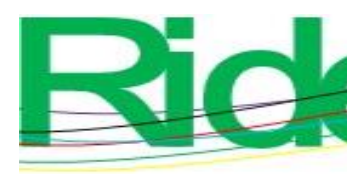

\section{Revista Iberoamericana para la Investigación y el Desarrollo Educativo ISSN 2007 - 7467}

\section{Resumo}

Para diagnosticar os hábitos que os novos universitários declaram ao desenvolver atividades relacionadas ao estudo, para identificar áreas de oportunidade que sirvam de referência ao empreender processos de redesenho curricular e planejamento acadêmico, essa contribuição investiga as práticas que Os alunos tendem a se apresentar diariamente quando se trata de estudar. Isso através da aplicação de um teste que identifica hábitos que os alunos têm quando estão matriculados em qualquer um dos cinco graus (Administração, Contabilidade, Gerenciamento de Turismo, Sistemas de Computador e Design de Software) oferecidos pela Faculdade de Contabilidade e Administração, Campus I ( FCA, CI) da Universidade Autônoma de Chiapas (Unach). O instrumento utilizado foi adaptado do proposto por Huidobro, Gutiérrez e Condemarín (2000). Assim, no final, consistiu em 39 itens com respostas dicotômicas (Sim / Não), através dos quais foram medidas oito dimensões: ambiente físico, estado físiológico, distribuição do tempo, leitura, técnicas de estudo, preparação de testes, concentração e Atitude em relação ao estudo. Por meio da plataforma eletrônica, o aplicativo foi autoadministrado e toda a população matriculada nos cinco graus de cada ano escolar participante foi coberta: um total de 1630 alunos. Em geral, os resultados mostram que, em média, cerca de $8(7,9)$ em cada 10 estudantes têm dificuldades em realizar atividades relacionadas à leitura e $6(5,9)$ em cada 10 relatam problemas com a distribuição do tempo. Da mesma forma, globalmente, em média, 23\% dos participantes obtêm ponderações aceitáveis em apenas duas das oito dimensões exploradas (embora essa média mostre diferenças significativas por grau); $26 \%$ entre três e quatro; $30 \%$ entre cinco e seis e apenas $21 \%$ declaram sete ou oito dimensões aceitáveis. Esses resultados podem ser usados como parâmetros de referência para projetar no futuro programas sistemáticos e diferenciados de intervenção acadêmica que permitam promover e melhorar a leitura, técnicas de distribuição de tempo, melhorar a concentração e criar as condições físicas propícias ao estudo, e mesmo para projetar programas sistemáticos de treinamento para professores.

Palavras-chave: educação, ensino superior, estudante universitário, processo de aprendizagem.

Fecha Recepción: Diciembre 2019

Fecha Aceptación: Julio 2020 


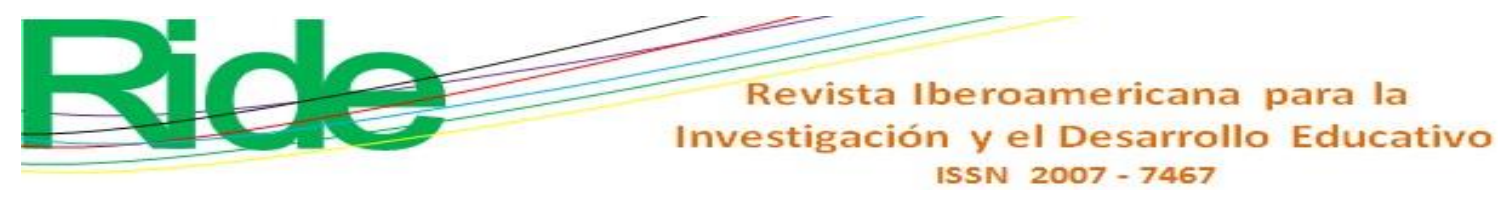

\section{Introducción}

Aun cuando existen evidencias de que la educación en los últimos años ha incrementado su cobertura y los grados de escolaridad promedio (Narro, Martuscelli y Barzana, 2012), persisten los problemas relacionados con la calidad. A las complicaciones que provoca la deserción y los índices de reprobación, en los últimos tiempos se les suman los resultados insuficientes que los estudiantes obtienen en las pruebas estandarizadas, especialmente en las competencias relacionadas con matemáticas, lectura y ciencias naturales (Ibarrola, 2012). Cifras de la Organización para la Cooperación y Desarrollo Económico [OCDE] (2017) señalan que en México de cada 100 alumnos que ingresan a la primaria solo 21 concluyen la universidad, cuatro estudian una maestría y solo uno llega al doctorado. De ahí que solo 17 \% de la población de entre 25 y 64 años haya cursado la educación superior, la cifra más baja entre los países miembros de la OCDE (2017), cuyo promedio es de $37 \%$.

Por su parte, en el diagnóstico que sobre la educación media superior realizó el Instituto Nacional para la Evaluación de la Educación [INEE] (2011) se detectó que quienes egresan de ese nivel educativo únicamente son capaces de realizar lecturas sencillas, sin lograr asociar la información de los textos con el conocimiento cotidiano y con serias deficiencias para utilizar a la lectura como herramienta de apoyo al aprendizaje, lo que puede ser la causa de eventuales problemas de aprovechamiento cuando se transita a la universidad, puesto que en la educación superior se suele exigir la lectura de textos que requieren, para su cabal entendimiento, que los lectores sean capaces de realizar inferencias, razonar respuestas, elaborar juicios y argumentar lógica y coherentemente (Vidal y Manríquez, 2016).

En este contexto, diagnosticar los hábitos de estudio que los estudiantes poseen al ingresar a la universidad representa un primer paso para instrumentar acciones de mejora continua que incidan en la práctica de estrategias efectivas de aprendizaje por parte de los propios discentes, pues si bien los problemas de deserción, abandono y rezago escolar en educación superior obedecen a múltiples factores (Asociación Nacional de Universidades e Instituciones de Educación Superior [Anuies], 2003; Castañeda y Ortega, 2004; Román, 2013), existe cierto convenio respecto a que, tratándose de problemas que afectan al rendimiento escolar, los perfiles inadecuados de ingreso, la falta de hábitos de estudio y la ausencia de procesos de autorregulación del aprendizaje se ubican dentro de las causas principales. $\mathrm{Y}$ en estas, la participación de los docentes universitarios juega un papel fundamental (Vidal y Manríquez, 2016). 


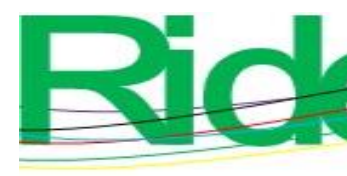

Revista Iberoamericana para la Investigación y el Desarrollo Educativo ISSN 2007 - 7467

Desde las consideraciones anteriores, en este trabajo se describen los métodos y estrategias que los estudiantes suelen realizar de forma cotidiana cuando se trata de cumplir con actividades de estudio. Unos y otras fueron determinados mediante la aplicación de un test que identifica hábitos que los estudiantes poseen, en específico, estudiantes que son matriculados en alguna de las cinco licenciaturas (Administración, Contaduría, Gestión Turística, Sistemas Computacionales y Diseño de Software) que ofrece la Facultad de Contaduría y Administración, Campus I (FCA, CI) de la Universidad Autónoma de Chiapas (Unach). Para ello, se analizó el comportamiento observado por estudiantes pertenecientes a cuatro cohortes, con el propósito de identificar tendencias y áreas de oportunidad que sirvan como insumo en el diseño de estrategias a incluir durante los procesos de actualización curricular y durante la planeación académica que desarrollan las autoridades educativas. Por llamarlo de alguna manera, los indicadores aquí socializados constituyen un referente a considerar en los procesos de rediseño curricular y en la planeación de estrategias orientadas a la mejora del aprendizaje de los estudiantes.

Cabe señalar que las prácticas declaradas por la propia universidad se tomaron como punto de partida, a saber: formar a profesionales de las áreas administrativas capaces de aprender por cuenta propia y de pensar de forma crítica y creativa; profesionales capaces de argumentar de forma oral y escrita aplicando un pensamiento sistémico y complejo en la construcción de conocimientos y toma de decisiones (Unach, 2015, 2016). Dicho perfil se ha construido a partir de las necesidades que tratará de solucionar el profesional en el potencial mercado ocupacional, en el análisis disciplinario que puede enriquecer las soluciones de los problemas del entorno y en la investigación de la propia universidad respecto a las actividades que realizará el futuro egresado (Díaz, 2011).

Para cumplir el propósito planteado, el documento se ha conformado bajo la estructura siguiente: enseguida a esta introducción, se plantea el objetivo general perseguido al realizar el proyecto y se enuncia el problema atendido. Posteriormente, se presenta una breve revisión de la literatura en torno al tópico en cuestión; allí se plasman conceptos centrales del marco de análisis sobre hábitos y prácticas de estudio, considerados como actividades y actitudes fundamentales a lo largo de toda la vida de las personas, pues, incluso al finalizar los estudios escolares y ser un profesional, es necesario recurrir sistemáticamente a aquellas estrategias y a aquellos hábitos que permitan enfrentar exitosamente los retos que se plantean en el ejercicio disciplinar; así, el alumno deberá desarrollar las competencias 


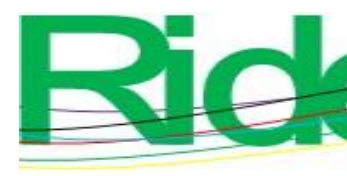

Revista Iberoamericana para la Investigación y el Desarrollo Educativo ISSN 2007-7467

necesarias para "seleccionar, organizar, reelaborar, jerarquizar, reflexionar y valorar críticamente la información para transformarla en genuino y relevante conocimiento personal y social" (Díaz y Hernández, 2010). También, a modo de colofón de este apartado, se describen algunos hallazgos relevantes encontrados en estudios similares.

Concluidos los referentes teóricos, se relata el método de colecta, análisis y procesamiento de datos, y se enfatiza en las características del instrumento empleado, así como las dimensiones exploradas, sin dejar de señalar las características generales de la población estudiada.

Más adelante, se presenta el apartado de discusión de resultados en donde se revelan las principales áreas de oportunidad detectadas como insumo a utilizar en los procesos de mejora continua; al final, las conclusiones recapitulan sobre el tema central y la importancia de considerar estos resultados como insumo básico de planeación de acciones académicas, impostergables para atender los desafíos que aquí se describen.

\section{Objetivo}

Diagnosticar los hábitos que los estudiantes universitarios de nuevo ingreso declaran cuando desarrollan actividades relacionadas con el estudio y con ello identificar las principales áreas de oportunidad que sirvan como referente al emprender procesos de rediseño curricular y de planeación académica capaces de contribuir significativamente al aprendizaje de los discentes.

\section{Problema}

Los hábitos de estudio son aquellos métodos y estrategias que una persona suele utilizar de forma cotidiana para abordar contenidos de aprendizaje, los cuales involucran esfuerzo, dedicación y disciplina, sin ignorar las expectativas y motivaciones que genera el deseo de aprender (Elizalde, 2017). En otras palabras, los hábitos de estudio son aquellos mecanismos que con regularidad utiliza un estudiante para asimilar unidades de aprendizaje, su disposición para evitar distracciones, su atención a materiales específicos y en general el esfuerzo que empeña a lo largo de todo el proceso de aprendizaje (Cartagena, 2008).

Esta práctica cotidiana de métodos y estrategias de aprendizaje involucra un conjunto de habilidades de trabajo intelectual que capacita al sujeto para una mejor y más fácil asimilación, transformación y creación de valores culturales. De acuerdo con Bedolla (2018), 


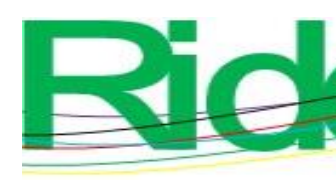

Revista Iberoamericana para la Investigación y el Desarrollo Educativo

ISSN $2007-7467$

las técnicas de estudio comprenden una serie de herramientas, esencialmente lógicas, que buscan mejorar el rendimiento de los alumnos y que apoyan los procesos de memorización, análisis y estudio. Mientras que desde la visión de la Secretaría de Educación Pública [SEP] (2014), los hábitos de estudio implican conductas o comportamientos repetitivos que los individuos ejecutan de forma continua y que resultan necesarios puesto que inciden en el reforzamiento del aprendizaje, facilitan el desarrollo de actitudes y permiten asumir responsabilidades, lo cual también da pie a mejorar las maneras de organización. De tal forma que es necesario fomentar en los alumnos competencias que involucren procesos de pensamiento y autorregulación eficientes, pues, independientemente del campo del conocimiento, una constante existe en los buenos estudiantes: no solo poseen una gran cantidad de conocimiento sobre la disciplina en cuestión, sino que lo asocian a estrategias cognitivas y autorregulatorias que apoyan ejecuciones exitosas, adquiridas con base en previas experiencias (Castañeda y Ortega, 2004). Así, producto de sus adecuados hábitos de estudio, una persona puede aprender con mayor rapidez y profundidad que otras.

Cabe señalar que estudiar de forma eficaz implica acciones que van más allá de la simple memorización; tiene que ver con identificar dónde y cómo recuperar la información requerida, acompañada de la capacidad para utilizarla inteligentemente (Bajwa, Gujjar, Shaheen y Ramzan, 2011).

Por otro lado, de acuerdo con Allgood, Risko, Álvarez y Fairbanks (2000, citados en Pérez, Valenzuela, Díaz, González y Núñez, 2013), la autorregulación y la actividad de estudio constituyen competencias que deberían aplicar y desarrollar los alumnos en la universidad, debido a que favorecen la autonomía, el aprendizaje activo y significativo para toda la vida. Y Monereo (2008) sugiere que las estrategias de aprendizaje debieran enseñarse de manera conjunta con las otras disciplinas involucradas en los planes de estudio, asumiendo que los universitarios que terminan con éxito sus estudios suelen estar motivados, con actitudes favorables hacia la licenciatura estudiada y con hábitos de estudio adecuados (Abarca, Gómez y Covarrubias, 2015). Es importante, por lo tanto, superar la idea de que el aprendizaje está limitado a contextos formales, institucionalizados y regulados, puesto que, por el contrario, se trata de una actividad que se desarrolla en esferas de la vida diaria, en aspectos personales, familiares y, por supuesto, profesionales (Castillo y Polanco, 2008). Así pues, la importancia de que los estudiantes puedan aprender a aprender reside en que esta acción sea permanente (Malander, 2014). 


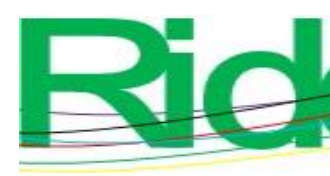

Revista Iberoamericana para la Investigación y el Desarrollo Educativo

ISSN $2007-7467$

Para lograr lo anterior, resulta indispensable identificar cuáles son las áreas de oportunidad que los universitarios de nuevo ingreso presentan cuando se trata de desarrollar actividades relacionadas con el estudio. Los resultados que se generen podrán utilizarse como referente cuando se desarrollen actividades de actualización y rediseño curricular y debieran servir como insumo en las acciones de planeación académica de la facultad.

Es decir, los resultados del estudio deberán constituir, junto con otros insumos, el punto de partida de las acciones a emprender para mejorar los procesos de aprendizaje de los universitarios, considerando que estos, cuando recién egresan de la educación media, suelen presentar problemas en la lectura: no la emplean como herramienta para la apropiación de nuevos contenidos y no logran asociar la información de los textos con el conocimiento cotidiano (INEE, 2011), lo que sin duda incide en el aprovechamiento a desarrollar en este nivel educativo, el cual demanda mejores competencias lectoras y mayor capacidad de argumentación lógica y respuestas coherentes (Vidal y Manríquez, 2016).

\section{Referentes}

La capacidad para estudiar no es algo con lo que se nace (como puede ser respirar), sino que se construye al incorporar un conjunto de técnicas que se aprenden a lo largo de la vida (Rowntree 2001). Como ya hemos mencionado, más allá de la conclusión de la formación escolarizada, los individuos habrán de enfrentar en el ejercicio profesional y a lo largo de la vida exigencias que demandan destrezas como leer rápido, comprender y recordar lo leído, organizar y distribuir el tiempo de forma adecuada, así como prepararse para resolver problemas cotidianos que precisan de hábitos de estudio eficaces, tal y como sucede en la vida universitaria. Es decir, se busca la autonomía del aprendizaje, término entendido como la facultad para tomar decisiones orientadas a regular el propio aprendizaje aproximándose a determinadas metas (Monereo, 2008). Así, aprender una estrategia no solo es saber ejecutar las operaciones de un procedimiento o técnica de aprendizaje, como pudieran ser la elaboración de esquemas, la técnica de subrayado, elaboración de resúmenes, mapas o diagramas; implica identificar cuándo y por qué deben emplearse.

No se alude a otra cosa, pues, que al aprendizaje efectivo. Un aprendizaje de estas características queda al descubierto cuando los estudiantes analizan las ventajas de un procedimiento respecto a otro, a partir de las características implícitas en la actividad a realizar, e incluso de la reflexión sobre por qué resulta apropiada determinada técnica o 


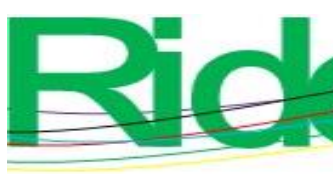

Revista Iberoamericana para la Investigación y el Desarrollo Educativo ISSN $2007-7467$

método, de ahí que se afirme que aprender es más que recibir respuestas del docente a preguntas que los estudiantes nunca se han formulado (García, Fonseca y Concha, 2015).

En ese sentido, Chain y Jácome (2007) afirman que quien desarrolla habilidades para resolver exámenes o hacer tareas, asiste con regularidad a bibliotecas, sabe consultar libros, elabora apuntes y acude a diversos profesores habrá construido un conjunto de rutinas de trabajo que potencialmente contribuirán al aprendizaje sistemático de contenidos e impactará en su desempeño cotidiano. Sin embargo, Cruz y Quiñones (2011, citados por Andrade et al., 2018) advierten que el éxito en el estudio no solo depende de la inteligencia y del esfuerzo, sino también de la eficiencia de los hábitos de estudio.

En el caso específico de la competencia lectora en estudiantes, Durán (2011) afirma que está relacionada con la calidad de la educación. Esto indudablemente plantea retos para las instituciones en el diseño y desarrollo de estrategias que la fortalezcan, a partir de considerar que su dominio conlleva un aprendizaje continuo que habrá de fortalecerse a lo largo de la vida académica y profesional (González, 2014).

Así, de acuerdo con Garrido (2014), “existe una relación directa, probada, entre el nivel de lectura de los estudiantes y su rendimiento escolar. Los mejores alumnos son mejores lectores que sus compañeros" (p. 148).

Y en palabras de Backhoff, Velasco y Peón (2013), es de esperarse que los universitarios, además de contar con la capacidad para comprender textos simples, desarrollen habilidades intelectuales para, entre otras cosas, discriminar ideas centrales, inferir conclusiones, resumir información, identificar ideas clave y argumentos de autores diferentes, sustentar una postura personal sobre temas específicos y expresarse por escrito de manera eficaz utilizando correctamente las normas del lenguaje.

Siguiendo con el hábito de la lectura, un trabajo de sistematización de 42 estudios respecto a niveles de competencia lectora, realizado por Manríquez (2015, citado en Vidal y Manríquez (2016), encontró que en 15 de ellos el nivel de comprensión lectora de los universitarios es bajo; tres muy bajo y tres se ubicaron en nivel regular. Asimismo, en dos encontraron un deficiente uso de estrategias lectoras y en otros dos se encontró que los estudiantes poseen pobres hábitos de lectura.

Por su parte, Hernández, Rodríguez y Vargas (2012), en un trabajo sobre hábitos de estudio y motivación para el aprendizaje en alumnos matriculados en carreras de ingeniería, encontraron que los estudiantes tienen problemas en la organización y planeación del estudio, 


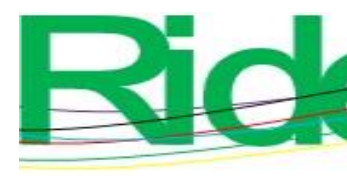

Revista Iberoamericana para la Investigación y el Desarrollo Educativo

ISSN $2007-7467$

las técnicas de memorización, la comprensión de lectura, el estudiar en casa y la carencia de estrategias para incrementar la motivación y la autoestima.

En esa misma línea, Arán y Ortega (2012) identificaron valores negativos (hábitos inadecuados) en relectura de textos, lectura en voz alta, toma de apuntes y en general en la capacidad de concentración en la lectura.

Respecto a estudios específicos de las áreas administrativas, Sarabia y Garizurieta (2006) realizaron en la Universidad Veracruzana (UV) una investigación sobre hábitos de estudio en estudiantes de la Facultad de Contaduría y Administración. Los participantes mostraron deficiencias en distribución y administración del tiempo, técnicas de lectura, capacidad de concentración y métodos de estudio. De igual forma, Lerma, Garrido y Hernández (2008) analizaron los hábitos de estudio en alumnos matriculados en carreras administrativas de la Universidad Juárez del Estado de Durango (UJED), cuya valoración global sobre hábitos de estudio aceptables fue de 68 (en escala del 1 al 100); las dimensiones vinculadas a la planeación, lectura y métodos de estudio obtuvieron bajas ponderaciones.

Ireta, González y Pérez (2008) realizaron un trabajo parecido en la Universidad Juárez Autónoma de Tabasco (UJAT) y detectaron que las áreas problemáticas en la práctica de estudios y actitudes para el aprendizaje en estudiantes de ciencias económico-administrativas estuvieron relacionadas con estrategias de aprendizaje y estudio para el logro, control y distribución del tiempo, técnicas de estudio y estrategias de examen.

Una investigación similar, aunque específicamente con estudiantes a punto de concluir la licenciatura en Administración de la Unach, realizado por Román, Sotelo y Aguilar (2016), concluyó que la dimensión Estado fisiológico era la que obtenía mejores ponderaciones, mientras que 7 de cada 10 encuestados presentaron deficiencias en las de Lectura y Distribución del tiempo.

Finalmente, se considera el estudio que evaluó la competencia de expresión escrita argumentativa que tienen los alumnos que concluyen la etapa de tronco común en la Facultad de Ciencias Administrativas y Sociales de la Universidad Autónoma de Baja California, (UABC), Campus Ensenada (Backhoff et al., 2013). Dicho trabajo, entre otros hallazgos, encontró que en general los estudiantes universitarios poseen habilidades muy pobres de expresión escrita argumentativa (la puntuación promedio en escala de 0 a 10 equivaldría a menos de 2.5), que solo 1 de cada 10 estudiantes realizan apuntes, diagramas o esquemas 


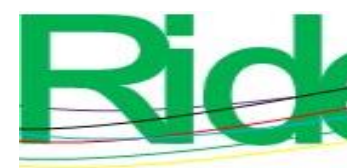

Revista Iberoamericana para la Investigación y el Desarrollo Educativo

ISSN $2007-7467$

para organizar la redacción de un artículo y que solo 3 de cada 100 hacen uso de vocabulario técnico adecuado.

\section{Método}

Basado en la clasificación ofrecida por Hernández, Fernández y Baptista (2014), el presente estudio es de alcance exploratorio y descriptivo. Para su desarrollo se observó el proceso básico planteado por Lara (2015), que sugiere las etapas de planeación de la investigación, recopilación de la información, procesamiento, interpretación y comunicación de las observaciones.

Los pasos desarrollados son similares a los observados en estudios realizados con anterioridad centrados en la identificación de hábitos de estudios con que egresan los estudiantes del área económico administrativa de la Unach (Román et al., 2016; Román, Gordillo y Franco, 2017); sin embargo, en este trabajo se presentan los resultados que corresponden en exclusiva a los alumnos de nuevo ingreso pertenecientes a cuatro cohortes.

Así, este trabajo es no experimental e involucra a estudiantes de nuevo ingreso de cuatro ciclos escolares: enero-junio y agosto-diciembre de 2018 y enero-junio y agostodiciembre de 2019, matriculados en los siguientes cinco programas de licenciatura: Contaduría (LC), Administración (LA), Gestión Turística (LGT), Sistemas Computacionales (LSC) e ingeniería en Desarrollo de Software (LIDS), ofertados en la FCA, CI de la Unach.

El instrumento empleado es una adaptación del test para identificar hábitos de estudio planteado por Huidobro, Gutiérrez y Condemarín (2000), traducido originalmente por Soto, con base en el denominado Inventario de hábitos de estudio creado por Wrenn, Eagle y Wright (citados en Urízar, 2012). Consta de 39 reactivos con respuestas dicotómicas (Sí / No), a través de las cuales se miden ocho dimensiones relacionadas con el ambiente físico, el estado fisiológico, la distribución de tiempo, la lectura, las técnicas de estudio, la preparación de pruebas, la concentración y la actitud hacia el estudio.

La aplicación del cuestionario fue autoadministrada. Se utilizó la plataforma electrónica con la que cuenta el cuerpo académico consolidado "Evaluación de procesos organizacionales" (Unach CA-137). La cobertura de la aplicación fue de toda la población, tal y como se señala en la tabla 1, la cual muestra por programa educativo el número de estudiantes de cada cohorte. 


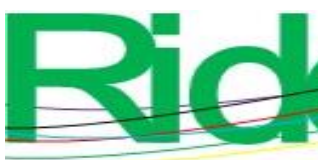

Revista Iberoamericana para la Investigación y el Desarrollo Educativo

ISSN $2007-7467$

Tabla 1. Cobertura alcanzada en aplicación del test para identificar hábitos de estudio en estudiantes de licenciatura de nuevo ingreso de la FCA, CI

\begin{tabular}{|l|c|c|c|c|c|c|c|}
\hline \multirow{2}{*}{ Programa educativo } & \multicolumn{3}{|c|}{ Número de estudiantes } & \multicolumn{3}{|c|}{ Estudiantes por ciclo escolar } \\
\cline { 3 - 8 } & \multicolumn{2}{|l|}{} & \multicolumn{2}{|c|}{$\mathbf{2 0 1 8}$} & \multicolumn{2}{c|}{2019} \\
\cline { 2 - 8 } & ABS & REL & ACUM & E-J & A-D & E-J & A-D \\
\hline LC & 480 & $29 \%$ & $29 \%$ & 123 & 135 & 112 & 110 \\
\hline LA & 453 & $28 \%$ & $57 \%$ & 124 & 119 & 104 & 106 \\
\hline LGT & 364 & $22 \%$ & $79 \%$ & 87 & 95 & 80 & 102 \\
\hline LSC & 226 & $14 \%$ & $93 \%$ & 26 & 85 & 20 & 95 \\
\hline LIDS & 107 & $7 \%$ & $100 \%$ & 17 & 32 & 0 & 58 \\
\hline Total & 1630 & $100 \%$ & & 377 & 466 & 316 & 471 \\
\hline
\end{tabular}

Fuente: Elaboración propia

Para concentrar la información y facilitar el análisis, se construyó, a través de tablas de frecuencias, una base de datos, donde el estadístico más representativo fue el promedio, representado en porcentajes. La información se generó a través de tablas de contingencias o cruzadas. Cabe señalar que en el caso de los datos obtenidos que presentan conjuntos de etiquetas ( Sí / No) se consideró que tienen un nivel de medición que corresponde a datos ordinales, que aun cuando "tienen valores relativos, se pueden ordenar o clasificar" (Lind, Marchal y Wathen, 2012, p. 11).

Para el procesamiento de los datos y la valoración de resultados, se observaron los criterios establecidos por los creadores del instrumento, los cuales establecen que para cada dimensión explorada los encuestados deben responder de la forma esperada en al menos $75 \%$ de las preguntas; con excepción de la dimensión Lectura, que requiere de 100 \% de respuestas acertadas.

Respecto a las variables analizadas, la tabla 2 muestra la conceptualización de estas, la forma en que se dimensionan, así como el número de indicadores y de preguntas que contempla cada una de ellas. 


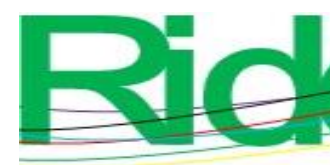

Revista Iberoamericana para la Investigación y el Desarrollo Educativo

ISSN 2007 - 7467

Tabla 2. Operacionalización de variables

\begin{tabular}{|c|c|c|c|}
\hline Conceptualización & Dimensiones & Indicadores & $\begin{array}{c}\text { Número } \\
\text { de } \\
\text { preguntas }\end{array}$ \\
\hline Ambiente físico & $\begin{array}{l}\text { Condiciones de } \\
\text { espacios } \\
\text { utilizados para } \\
\text { estudiar (lugar) y } \\
\text { el entorno que } \\
\text { prevalece al } \\
\text { estudiar. }\end{array}$ & $\begin{array}{l}\text { Estudio con radio y televisión } \\
\text { prendida o conversando con } \\
\text { personas en el mismo cuarto. } \\
\text { Lugar definido para estudiar. } \\
\text { Estudio en cama acostado. } \\
\text { Estudio con interrupciones por el } \\
\text { timbre, llamadas telefónicas y } \\
\text { visitas que atender. }\end{array}$ & 4 \\
\hline Estado fisiológico & $\begin{array}{l}\text { Condiciones } \\
\text { físicas y de salud } \\
\text { que prevalecen } \\
\text { cuando se realizan } \\
\text { actividades de } \\
\text { estudio. }\end{array}$ & $\begin{array}{l}\text { Cansancio o distracción para } \\
\text { estudiar con ganas. Dificultad } \\
\text { para levantarse e ir a la escuela. } \\
\text { Desánimo al estudiar. Nervios y } \\
\text { temor al contestar } \\
\text { interrogatorios. }\end{array}$ & 4 \\
\hline $\begin{array}{l}\text { Distribución de } \\
\text { tiempo }\end{array}$ & $\begin{array}{l}\text { Actividades de } \\
\text { organización y } \\
\text { distribución del } \\
\text { tiempo destinado } \\
\text { al estudio. }\end{array}$ & $\begin{array}{l}\text { Horario definido para estudiar. } \\
\text { Tiempo necesario y suficiente } \\
\text { para estudiar todas las materias. } \\
\text { Preparación de pruebas y } \\
\text { trabajos con anticipación. } \\
\text { Pérdida de tiempo durante el día, } \\
\text { dejando el estudio para la noche. } \\
\text { Tiempo dedicado al estudio por } \\
\text { día. }\end{array}$ & 5 \\
\hline Lectura & $\begin{array}{l}\text { Condiciones } \\
\text { cognitivas que } \\
\text { permiten analizar, } \\
\text { interpretar, } \\
\text { comprender y } \\
\text { sintetizar las ideas } \\
\text { leídas. }\end{array}$ & $\begin{array}{l}\text { Lectura de varias veces del texto, } \\
\text { pues las palabras no tienen } \\
\text { significado la primera vez. } \\
\text { Revisión de lo que se encuentra } \\
\text { dudoso, sin avanzar hasta } \\
\text { entender. Repetición de ideas } \\
\text { importantes, subrayado de ideas } \\
\text { principales. Dificultad para } \\
\text { encontrar ideas importantes. } \\
\text { Equivocaciones constantes, } \\
\text { cambio de palabras, significado y } \\
\text { puntuación. }\end{array}$ & 5 \\
\hline Técnicas de estudio & $\begin{array}{l}\text { Empleo de } \\
\text { esquemas o } \\
\text { estrategias para el } \\
\text { abordaje del } \\
\text { objeto de estudio, } \\
\text { que se consideren } \\
\text { útiles para facilitar } \\
\text { la comprensión de }\end{array}$ & $\begin{array}{l}\text { Revisión general: análisis de } \\
\text { títulos, subtítulos, índice, } \\
\text { resumen, para obtener ideas } \\
\text { generales. Estudio activo, con la } \\
\text { elaboración de apuntes, resumen, } \\
\text { subrayado o esquemas. Relación } \\
\text { entre lo aprendido en una materia } \\
\text { con otras. Conclusión de una }\end{array}$ & 6 \\
\hline
\end{tabular}




\begin{tabular}{|l|l|l|c|}
\hline & $\begin{array}{l}\text { los temas de } \\
\text { estudio. }\end{array}$ & $\begin{array}{l}\text { tarea antes de iniciar otra. } \\
\text { Privilegio de la memorización, } \\
\text { sin entender significado. Repaso } \\
\text { periódico de lo estudiado. }\end{array}$ & \\
\hline $\begin{array}{l}\text { Preparación de } \\
\text { pruebas }\end{array}$ & $\begin{array}{l}\text { Refiere los } \\
\text { procedimientos } \\
\text { adoptados para } \\
\text { prepararse antes } \\
\text { de una evaluación, } \\
\text { anticipando los } \\
\text { la prueba. Información de todo el } \\
\text { contenido a evaluar y material } \\
\text { necesario para estudiar. Atención } \\
\text { posibles. }\end{array}$ & $\begin{array}{l}\text { inicial de instrucciones y } \\
\text { preguntas antes de iniciar con el } \\
\text { estudio. Nervios que impiden } \\
\text { demostrar lo que se conoce. } \\
\text { Estudio solo para las pruebas. }\end{array}$ & 5 \\
\hline Concentración & $\begin{array}{l}\text { Mecanismos } \\
\text { empleados que } \\
\text { contribuyen en los } \\
\text { estudiantes a } \\
\text { enfocar la } \\
\text { atención en } \\
\text { actividades de } \\
\text { estudio. }\end{array}$ & $\begin{array}{l}\text { Estudio y realización de otras } \\
\text { actividades (pararse, caminar). } \\
\text { Dificultad para mantener } \\
\text { atención y para comprender. } \\
\text { Distracción fácil ante ruidos o } \\
\text { situaciones imprevistas. } \\
\text { Mantener atención en clases. } \\
\text { Inicio de otras actividades sin } \\
\text { concluir la anterior. }\end{array}$ & 5 \\
\hline $\begin{array}{l}\text { Actitud hacia el } \\
\text { estudio }\end{array}$ & $\begin{array}{l}\text { Prejuicios } \\
\text { formulados con } \\
\text { base en los sujetos } \\
\text { que intervienen en } \\
\text { el proceso } \\
\text { educativo y el rol } \\
\text { del sujeto que } \\
\text { aprende en los } \\
\text { diversos } \\
\text { escenarios áulicos. }\end{array}$ & $\begin{array}{l}\text { relacionadas con el estudio, lo } \\
\text { que perjudica el rendimiento. } \\
\text { Aburrimiento al estudiar. } \\
\text { Contribución al desorden en } \\
\text { clases. Participación activa en el } \\
\text { trabajo en clases. Participación } \\
\text { activa en el trabajo del grupo y } \\
\text { expresión de opinión. }\end{array}$ & \\
\end{tabular}

Fuente: Elaboración propia con base en Huidobro et al. (2000)

Estas ocho variables son exploradas a través de 39 preguntas dicotómicas, en donde los encuestados expresan con libertad su acuerdo o desacuerdo con la afirmación que se les plantea. Aquí se parte de la premisa de que un estudiante que domina las ocho dimensiones sobre las que gira el test demostrará los hábitos de estudio que ha construido a lo largo de su instrucción escolar. De tal forma que aun cuando concluya los procesos escolarizados de formación, los hábitos adquiridos permitirán desarrollar actitudes favorables para el estudio y el aprendizaje para toda la vida, considerando las condiciones físicas en las que estudia, el estado de salud que facilita el aprendizaje, la habilidad para distribuir el tiempo dedicado al estudio, las habilidades de lectura y comprensión, la aplicación de técnicas de estudio al involucrarse en procesos de aprendizaje, los procedimientos utilizados para anticiparse a las 

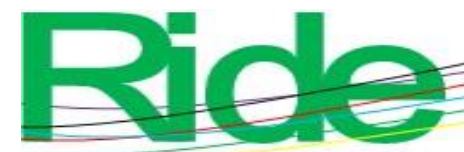

Revista Iberoamericana para la Investigación y el Desarrollo Educativo ISSN 2007 - 7467

pruebas a resolver, la capacidad de concentración y en general el mantenimiento de una actitud positiva hacia el estudio.

\section{Resultados}

Como se ha venido comentado, los estudiantes que participan en el estudio están matriculados en las cinco licenciaturas que ofrece la FCA, CI de la Unach. En cuanto a la distribución, los de LC tuvieron mayor porcentaje de participación en el estudio al conformar $29 \%$ del universo, seguidos de LA (28 \%), LGT (22\%), LSC (14\%) y LIDS (7 \%).

Los resultados que corresponden a la LC muestran que las dimensiones en donde los estudiantes obtuvieron mejores ponderaciones (arriba de $60 \%$ ) se relacionan con la actitud hacia el estudio (76\%), las técnicas de estudio (68\%), la preparación de pruebas (66\%), el estado fisiológico (65\%) y la concentración (62\%); en contraste, las principales áreas de oportunidad están relacionadas con la lectura (78\%), distribución del tiempo (48\%) y ambiente físico (45\%). Estos resultados se describen en la tabla 3.

Bajo el criterio de considerar como aceptable cualquier porcentaje igual o superior a $60 \%$, los estudiantes de esta licenciatura presentan hábitos aceptables en cinco de las ocho dimensiones exploradas y las áreas de oportunidad son: lectura, distribución del tiempo y ambiente físico. 


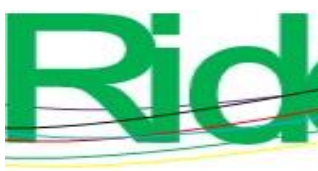

Revista Iberoamericana para la Investigación y el Desarrollo Educativo

ISSN $2007-7467$

Tabla 3. Resultados por dimensión y ciclo escolar de estudiantes de LC

\begin{tabular}{|c|c|c|c|c|c|c|c|c|c|c|c|c|c|c|c|c|c|c|c|c|}
\hline \multirow[t]{4}{*}{ D } & \multicolumn{8}{|c|}{2018} & \multicolumn{8}{|c|}{2019} & \multirow{2}{*}{\multicolumn{4}{|c|}{$\begin{array}{c}\text { Acumulado } \\
\text { cuatro } \\
\text { cohortes }\end{array}$}} \\
\hline & \multicolumn{4}{|c|}{ Ene-Jun } & \multicolumn{4}{|c|}{ Ago-Dic } & \multicolumn{4}{|c|}{ Ene-Jun } & \multicolumn{4}{|c|}{ Ago-Dic } & & & & \\
\hline & \multicolumn{2}{|c|}{ AD } & \multicolumn{2}{|c|}{ IN } & \multicolumn{2}{|c|}{ AD } & \multicolumn{2}{|c|}{ IN } & \multicolumn{2}{|c|}{ AD } & \multicolumn{2}{|c|}{ IN } & \multicolumn{2}{|c|}{ AD } & \multicolumn{2}{|c|}{ IN } & \multicolumn{2}{|c|}{ AD } & \multicolumn{2}{|c|}{ IN } \\
\hline & $\begin{array}{l}\mathbf{A} \\
\mathbf{b}\end{array}$ & $\mathbf{R}$ & $\begin{array}{l}\mathbf{A} \\
\mathbf{b}\end{array}$ & $\mathbf{R}$ & $\begin{array}{l}\mathbf{A} \\
\mathbf{b}\end{array}$ & $\mathbf{R}$ & $\begin{array}{l}\mathbf{A} \\
\mathbf{b}\end{array}$ & $\mathbf{R}$ & $\begin{array}{l}\mathbf{A} \\
\mathbf{b}\end{array}$ & $\mathbf{R}$ & $\begin{array}{l}\mathbf{A} \\
\mathbf{b}\end{array}$ & $\mathbf{R}$ & $\begin{array}{l}\text { A } \\
\text { b }\end{array}$ & $\mathbf{R}$ & $\begin{array}{l}\mathbf{A} \\
\mathbf{b}\end{array}$ & $\mathbf{R}$ & $\begin{array}{l}\mathbf{A} \\
\mathbf{b}\end{array}$ & $\mathbf{R}$ & $\begin{array}{l}\mathbf{A} \\
\mathbf{b}\end{array}$ & $\mathbf{R}$ \\
\hline $\mathbf{A}$ & 6 & 5 & 5 & 4 & 70 & 5 & 65 & 4 & 6 & 5 & 46 & 4 & 5 & 5 & 5 & 4 & 20 & 5 & 1 & 4 \\
\hline $\mathbf{F}$ & 9 & 6 & 4 & 4 & & 2 & & 8 & 6 & 9 & & 1 & 7 & 2 & 3 & 8 & 5 & 5 & $\begin{array}{l}6 \\
5\end{array}$ & 5 \\
\hline $\mathbf{E}$ & 7 & 6 & 4 & 3 & 84 & 6 & 51 & 3 & 8 & 7 & 32 & 2 & 6 & 5 & 4 & 4 & 23 & 6 & 1 & 3 \\
\hline $\mathbf{F}$ & 5 & 1 & 8 & 9 & & 2 & & 8 & 0 & 1 & & 9 & 2 & 6 & 8 & 4 & 9 & 5 & $\begin{array}{l}3 \\
1\end{array}$ & 5 \\
\hline D & 6 & 5 & 5 & 4 & 66 & 4 & 69 & 5 & 6 & 5 & 51 & 4 & 5 & 4 & 5 & 5 & 19 & 5 & 1 & 4 \\
\hline & 6 & 4 & 7 & 6 & & 9 & & 1 & 1 & 4 & & 6 & 1 & 6 & 9 & 4 & 3 & 2 & $\begin{array}{l}7 \\
7\end{array}$ & 8 \\
\hline $\mathbf{L}$ & 3 & 2 & 9 & 7 & 23 & 1 & 11 & 8 & 2 & 2 & 85 & 7 & 2 & 1 & 9 & 8 & 83 & 2 & 2 & 7 \\
\hline & 3 & 7 & 0 & 3 & & 7 & 2 & 3 & 7 & 4 & & 6 & 0 & 8 & 0 & 2 & & 2 & $\begin{array}{l}8 \\
7\end{array}$ & 8 \\
\hline $\mathbf{T}$ & 8 & 7 & 3 & 2 & 80 & 5 & 55 & 4 & 8 & 7 & 28 & 2 & 8 & 7 & 2 & 2 & 25 & 6 & 1 & 3 \\
\hline $\mathbf{E}$ & 7 & 1 & 6 & 9 & & 9 & & 1 & 4 & 5 & & 5 & 5 & 7 & 5 & 3 & 1 & 8 & $\begin{array}{l}1 \\
9\end{array}$ & 2 \\
\hline $\mathbf{P}$ & 7 & 6 & 4 & 3 & 85 & 6 & 50 & 3 & 8 & 7 & 32 & 2 & 7 & 6 & 3 & 3 & 24 & 6 & 1 & 3 \\
\hline $\mathbf{P}$ & 8 & 3 & 5 & 7 & & 3 & & 7 & 0 & 1 & & 9 & 1 & 5 & 9 & 5 & 3 & 6 & $\begin{array}{l}2 \\
7\end{array}$ & 4 \\
\hline $\mathrm{C}$ & 8 & 7 & 3 & 3 & 74 & 5 & 61 & 4 & 7 & 6 & 41 & 3 & 6 & 5 & 5 & 4 & 23 & 6 & 1 & 3 \\
\hline & 6 & 0 & 7 & 0 & & 5 & & 5 & 1 & 3 & & 7 & 0 & 5 & 0 & 5 & 1 & 2 & $\begin{array}{l}3 \\
9\end{array}$ & 8 \\
\hline $\mathbf{A}$ & 9 & 8 & 2 & 2 & 94 & 7 & 41 & 3 & 8 & 7 & 24 & 2 & 8 & 7 & 3 & 2 & 28 & 7 & 8 & 2 \\
\hline & 9 & 0 & 4 & 0 & & 0 & & 0 & 8 & 9 & & 1 & 0 & 3 & 0 & 7 & 1 & 6 & 9 & 4 \\
\hline
\end{tabular}

Notas: $\mathrm{D}=$ Dimensiones; $\mathrm{AF}=\mathrm{Ambiente}$ físico; $\mathrm{EF}=$ Estado fisiológico; $\mathrm{D}=$ Distribución

del tiempo; $\mathrm{L}=$ Lectura $; \mathrm{TE}=$ Técnicas de estudio; $\mathrm{PP}=$ Preparación de pruebas; $\mathrm{C}=$

Concentración; $\mathrm{A}=$ Actitud hacia el estudio. $\mathrm{AD}=$ Hábitos adecuados; $\mathrm{IN}=$ Hábitos inadecuados; $\mathrm{Ab}=$ Valor absoluto; $\mathrm{R}=$ Valor relativo.

Fuente: Elaboración propia

Los hábitos declarados por los estudiantes de LA se muestran en la tabla 4. Allí se puede apreciar que las dimensiones en donde los estudiantes obtienen mejores ponderaciones son Actitud hacia el estudio (78 \%), Técnicas de estudio y Preparación de pruebas (ambas con $67 \%$ ), Estado fisiológico (66 \%) y Concentración (61 \%); en contraposición, y al igual que en el caso de los estudiantes de LC, las principales áreas de oportunidad se relacionan con la lectura (70\%), la distribución del tiempo (53\%) y el ambiente físico (48\%). 


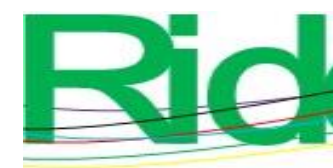

Revista Iberoamericana para la Investigación y el Desarrollo Educativo

ISSN 2007 - 7467

De tal forma que, siguiendo el mismo criterio de considerar hábitos aceptables aquellos que alcanza $60 \%$ o más, las áreas de oportunidad son las mismas que se presentan en LC.

Tabla 4. Resultados por dimensión y ciclo escolar de estudiantes de LA

\begin{tabular}{|c|c|c|c|c|c|c|c|c|c|c|c|c|c|c|c|c|c|c|c|c|}
\hline \multirow[t]{4}{*}{ D } & \multicolumn{8}{|c|}{2018} & \multicolumn{8}{|c|}{2019} & \multirow{2}{*}{\multicolumn{4}{|c|}{$\begin{array}{c}\text { Acumulado } \\
\text { cuatro } \\
\text { cohortes } \\
\end{array}$}} \\
\hline & \multicolumn{4}{|c|}{ Ene-Jun } & \multicolumn{4}{|c|}{ Ago-Dic } & \multicolumn{4}{|c|}{ Ene-Jun } & \multicolumn{4}{|c|}{ Ago-Dic } & & & & \\
\hline & \multicolumn{2}{|c|}{ AD } & \multicolumn{2}{|c|}{ IN } & \multicolumn{2}{|c|}{ AD } & \multicolumn{2}{|c|}{ IN } & \multicolumn{2}{|c|}{ AD } & \multicolumn{2}{|c|}{ IN } & \multicolumn{2}{|c|}{ AD } & \multicolumn{2}{|c|}{ IN } & \multicolumn{2}{|c|}{ AD } & \multicolumn{2}{|c|}{ IN } \\
\hline & $\begin{array}{l}\mathbf{A} \\
\mathbf{b}\end{array}$ & $\mathbf{R}$ & $\begin{array}{l}\mathbf{A} \\
\mathbf{b}\end{array}$ & $\mathbf{R}$ & $\begin{array}{l}\mathbf{A} \\
\mathbf{b}\end{array}$ & $\mathbf{R}$ & $\begin{array}{l}\mathbf{A} \\
\mathbf{b}\end{array}$ & $\mathbf{R}$ & $\begin{array}{l}\mathbf{A} \\
\mathbf{b}\end{array}$ & $\mathbf{R}$ & $\begin{array}{l}\mathbf{A} \\
\mathbf{b}\end{array}$ & $\mathbf{R}$ & $\begin{array}{l}\mathbf{A} \\
\mathbf{b}\end{array}$ & $\mathbf{R}$ & $\begin{array}{l}\mathbf{A} \\
\mathbf{b}\end{array}$ & $\mathbf{R}$ & $\begin{array}{l}\mathbf{A} \\
\mathbf{b}\end{array}$ & $\mathbf{R}$ & $\begin{array}{l}\mathbf{A} \\
\mathbf{b}\end{array}$ & $\mathbf{R}$ \\
\hline $\mathbf{A}$ & 7 & 5 & 5 & 4 & 63 & 5 & 56 & 4 & 5 & 5 & 52 & 5 & 4 & 4 & 5 & 5 & 23 & 5 & 2 & 4 \\
\hline $\mathbf{F}$ & 2 & 8 & 2 & 2 & & 3 & & 7 & 2 & 0 & & 0 & 9 & 6 & 7 & 4 & 6 & 2 & $\begin{array}{l}1 \\
7\end{array}$ & 8 \\
\hline $\mathbf{E}$ & 9 & 7 & 3 & 2 & 81 & 6 & 38 & 3 & 6 & 6 & 35 & 3 & 5 & 5 & 4 & 4 & 30 & 6 & 1 & 3 \\
\hline $\mathbf{F}$ & 1 & 3 & 3 & 7 & & 8 & & 2 & 9 & 6 & & 4 & 9 & 6 & 7 & 4 & 0 & 6 & $\begin{array}{l}5 \\
3\end{array}$ & 4 \\
\hline D & 6 & 5 & 5 & 4 & 59 & 5 & 60 & 5 & 4 & 4 & 55 & 5 & 3 & 3 & 6 & 6 & 21 & 4 & 2 & 5 \\
\hline & 8 & 5 & 6 & 5 & & 0 & & 0 & 9 & 7 & & 3 & 8 & 6 & 8 & 4 & 4 & 7 & $\begin{array}{l}3 \\
9\end{array}$ & 3 \\
\hline $\mathbf{L}$ & 3 & 3 & 8 & 6 & 33 & 2 & 86 & 7 & 2 & 2 & 76 & 7 & 3 & 3 & 7 & 6 & 13 & 3 & 3 & 7 \\
\hline & 9 & 1 & 5 & 9 & & 8 & & 2 & 8 & 7 & & 3 & 4 & 2 & 2 & 8 & 4 & 0 & $\begin{array}{l}1 \\
9\end{array}$ & 0 \\
\hline $\mathbf{T}$ & 8 & 6 & 4 & 3 & 86 & 7 & 33 & 2 & 7 & 7 & 30 & 2 & 6 & 6 & 4 & 4 & 30 & 6 & 1 & 3 \\
\hline $\mathbf{E}$ & 1 & 5 & 3 & 5 & & 2 & & 8 & 4 & 1 & & 9 & 4 & 0 & 2 & 0 & 5 & 7 & $\begin{array}{l}4 \\
8\end{array}$ & 3 \\
\hline $\mathbf{P}$ & 8 & 7 & 3 & 2 & 85 & 7 & 34 & 2 & 6 & 6 & 37 & 3 & 6 & 5 & 4 & 4 & 30 & 6 & 1 & 3 \\
\hline $\mathbf{P}$ & 9 & 2 & 5 & 8 & & 1 & & 9 & 7 & 4 & & 6 & 2 & 8 & 4 & 2 & 3 & 7 & $\begin{array}{l}5 \\
0\end{array}$ & 3 \\
\hline $\mathbf{C}$ & 8 & 6 & 4 & 3 & 72 & 6 & 47 & 3 & 6 & 6 & 39 & 3 & 5 & 5 & 4 & 4 & 27 & 6 & 1 & 3 \\
\hline & 1 & 5 & 3 & 5 & & 1 & & 9 & 5 & 3 & & 7 & 7 & 4 & 9 & 6 & 5 & 1 & $\begin{array}{l}7 \\
8\end{array}$ & 9 \\
\hline $\mathbf{A}$ & 9 & 7 & 2 & 2 & 98 & 8 & 21 & 1 & 8 & 7 & 22 & 2 & 7 & 7 & 2 & 2 & 35 & 7 & 9 & 2 \\
\hline & 6 & 7 & 8 & 3 & & 2 & & 8 & 2 & 9 & & 1 & 9 & 5 & 7 & 5 & 5 & 8 & 8 & 2 \\
\hline
\end{tabular}

Notas: $\mathrm{D}=$ Dimensiones; $\mathrm{AF}=\mathrm{Ambiente}$ físico; $\mathrm{EF}=$ Estado fisiológico; $\mathrm{D}=$ Distribución

del tiempo; $\mathrm{L}=$ Lectura; $\mathrm{TE}=$ Técnicas de estudio; $\mathrm{PP}=$ Preparación de pruebas; $\mathrm{C}=$ Concentración; A = Actitud hacia el estudio. AD = Hábitos adecuados; IN = Hábitos inadecuados; $\mathrm{Ab}=$ Valor absoluto; $\mathrm{R}=$ Valor relativo.

Fuente: Elaboración propia

Respecto a los resultados de la licenciatura en LGT, en la tabla 5 se observa que, con pequeños matices, el comportamiento es similar al obtenido en LC y LA, ya que los porcentajes de hábitos aceptables tienen que ver con las mismas cinco dimensiones: Actitud 


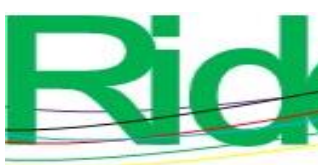

Revista Iberoamericana para la Investigación y el Desarrollo Educativo

ISSN 2007 - 7467

hacia el estudio (83\%), Técnicas de estudio (68\%), Estado fisiológico (65\%), Concentración (62\%) y Preparación de pruebas (61\%); mientras que las principales áreas de oportunidad se relacionan con la lectura (75\%), la distribución del tiempo (52\%) y el ambiente físico (45\%).

Tabla 5. Resultados por dimensión y ciclo escolar de estudiantes de LGT

\begin{tabular}{|c|c|c|c|c|c|c|c|c|c|c|c|c|c|c|c|c|c|c|c|c|}
\hline \multirow[t]{4}{*}{ D } & \multicolumn{8}{|c|}{2018} & \multicolumn{8}{|c|}{2019} & \multirow{2}{*}{\multicolumn{4}{|c|}{$\begin{array}{c}\text { Acumulado } \\
\text { cuatro } \\
\text { cohortes }\end{array}$}} \\
\hline & \multicolumn{4}{|c|}{ Ene-Jun } & \multicolumn{4}{|c|}{ Ago-Dic } & \multicolumn{4}{|c|}{ Ene-Jun } & \multicolumn{4}{|c|}{ Ago-Dic } & & & & \\
\hline & \multicolumn{2}{|c|}{ AD } & \multicolumn{2}{|c|}{ IN } & \multicolumn{2}{|c|}{ AD } & \multicolumn{2}{|c|}{ IN } & \multicolumn{2}{|c|}{ AD } & \multicolumn{2}{|c|}{ IN } & \multicolumn{2}{|c|}{ AD } & \multicolumn{2}{|c|}{ IN } & \multicolumn{2}{|c|}{ AD } & \multicolumn{2}{|c|}{ IN } \\
\hline & $\begin{array}{l}\mathbf{A} \\
\mathbf{b}\end{array}$ & $\mathbf{R}$ & $\begin{array}{l}\mathbf{A} \\
\mathbf{b}\end{array}$ & $\mathbf{R}$ & $\begin{array}{l}\mathbf{A} \\
\mathbf{b}\end{array}$ & $\mathbf{R}$ & $\begin{array}{l}\mathbf{A} \\
\mathbf{b}\end{array}$ & $\mathbf{R}$ & $\begin{array}{l}\mathbf{A} \\
\mathbf{b}\end{array}$ & $\mathbf{R}$ & $\begin{array}{l}\mathbf{A} \\
\mathbf{b}\end{array}$ & $\mathbf{R}$ & $\begin{array}{l}\mathbf{A} \\
\mathbf{b}\end{array}$ & $\mathbf{R}$ & $\begin{array}{l}\mathbf{A} \\
\mathbf{b}\end{array}$ & $\mathbf{R}$ & $\begin{array}{l}\mathbf{A} \\
\mathbf{b}\end{array}$ & $\mathbf{R}$ & $\begin{array}{l}\mathbf{A} \\
\mathbf{b}\end{array}$ & $\mathbf{R}$ \\
\hline $\mathbf{A}$ & 4 & 5 & 3 & 4 & 52 & 5 & 43 & 4 & 4 & 5 & 35 & 4 & 5 & 5 & 4 & 4 & 20 & 5 & 1 & 4 \\
\hline $\mathbf{F}$ & 8 & 5 & 9 & 5 & & 5 & & 5 & 5 & 6 & & 4 & 5 & 4 & 7 & 6 & 0 & 5 & $\begin{array}{l}6 \\
4\end{array}$ & 5 \\
\hline $\mathbf{E}$ & 6 & 7 & 2 & 2 & 65 & 6 & 30 & 3 & 5 & 6 & 29 & 3 & 5 & 5 & 4 & 4 & 23 & 6 & 1 & 3 \\
\hline $\mathbf{F}$ & 4 & 4 & 3 & 6 & & 8 & & 2 & 1 & 4 & & 6 & 7 & 6 & 5 & 4 & 7 & 5 & $\begin{array}{l}2 \\
7\end{array}$ & 5 \\
\hline $\mathbf{D}$ & 5 & 6 & 3 & 3 & 44 & 4 & 51 & 5 & 3 & 4 & 43 & 5 & 4 & 4 & 6 & 6 & 17 & 4 & 1 & 5 \\
\hline & 3 & 1 & 4 & 9 & & 6 & & 4 & 7 & 6 & & 4 & 1 & 0 & 1 & 0 & 5 & 8 & $\begin{array}{l}8 \\
9\end{array}$ & 2 \\
\hline $\mathbf{L}$ & 2 & 2 & 6 & 7 & 31 & 3 & 64 & 6 & 1 & 2 & 62 & 7 & 1 & 1 & 8 & 8 & 91 & 2 & 2 & 7 \\
\hline & 3 & 6 & 4 & 4 & & 3 & & 7 & 8 & 3 & & 7 & 9 & 9 & 3 & 1 & & 5 & $\begin{array}{l}7 \\
3 \\
\end{array}$ & 5 \\
\hline $\mathbf{T}$ & 5 & 6 & 3 & 3 & 74 & 7 & 21 & 2 & 5 & 6 & 25 & 3 & 6 & 6 & 4 & 4 & 24 & 6 & 1 & 3 \\
\hline $\mathbf{E}$ & 7 & 6 & 0 & 4 & & 8 & & 2 & 5 & 9 & & 1 & 1 & 0 & 1 & 0 & 7 & 8 & $\begin{array}{l}1 \\
7\end{array}$ & 2 \\
\hline $\mathbf{P}$ & 6 & 7 & 2 & 2 & 56 & 5 & 39 & 4 & 4 & 6 & 32 & 4 & 5 & 5 & 5 & 5 & 22 & 6 & 1 & 3 \\
\hline $\mathbf{P}$ & 7 & 7 & 0 & 3 & & 9 & & 1 & 8 & 0 & & 0 & 1 & 0 & 1 & 0 & 2 & 1 & $\begin{array}{l}4 \\
2 \\
\end{array}$ & 9 \\
\hline $\mathbf{C}$ & 6 & 7 & 2 & 2 & 68 & 7 & 27 & 2 & 4 & 5 & 38 & 4 & 5 & 5 & 5 & 5 & 22 & 6 & 1 & 3 \\
\hline & 4 & 4 & 3 & 6 & & 2 & & 8 & 2 & 3 & & 7 & 1 & 0 & 1 & 0 & 5 & 2 & $\begin{array}{l}3 \\
9\end{array}$ & 8 \\
\hline $\mathbf{A}$ & 8 & 9 & 5 & 6 & 78 & 8 & 17 & 1 & 6 & 8 & 11 & 1 & 7 & 7 & 2 & 2 & 30 & 8 & 6 & 1 \\
\hline & 2 & 4 & & & & 2 & & 8 & 9 & 6 & & 4 & 4 & 3 & 8 & 7 & 3 & 3 & 1 & 7 \\
\hline
\end{tabular}

Notas: $\mathrm{D}=$ Dimensiones; $\mathrm{AF}=\mathrm{Ambiente}$ físico; $\mathrm{EF}=$ Estado fisiológico; $\mathrm{D}=$ Distribución del tiempo; $\mathrm{L}=$ Lectura; $\mathrm{TE}=$ Técnicas de estudio; $\mathrm{PP}=$ Preparación de pruebas; $\mathrm{C}=$ Concentración; A = Actitud hacia el estudio. AD = Hábitos adecuados; IN = Hábitos inadecuados; $\mathrm{Ab}=$ Valor absoluto; $\mathrm{R}=$ Valor relativo.

Fuente: Elaboración propia 

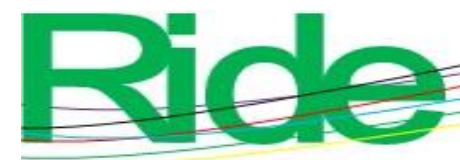

Los resultados de LSC, presentados en la tabla 6, describen un comportamiento diferente respecto a las tres licenciaturas anteriores, pues solo la dimensión Actitud hacia el estudio alcanza una ponderación superior a $60 \%$, al promediar $69 \%$; en tanto que las relacionadas con preparación de pruebas y técnicas de estudio (ambas con $51 \%$ ) y ambiente físico (53\%) apenas sobrepasan a la mayoría de los participantes. Todavía más significativo es que en las otras cuatro la proporción de hábitos inadecuados alcanza al menos a la mitad de los estudiantes: Lectura (87\%), Distribución del tiempo (65 \%), Concentración (56 \%) y Estado fisiológico (50\%). De manera que comparadas con las licenciaturas anteriores (LC, LA y LGT), además de las tres áreas de oportunidad ya detectadas, se agregan las relacionadas con el estado fisiológico, la preparación de pruebas, las técnicas de estudio y la concentración; es decir, existe un considerable margen de mejora en siete de las ocho dimensiones analizadas. 


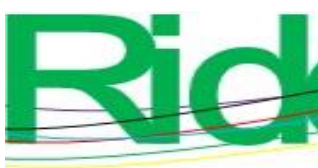

Revista lberoamericana para la Investigación y el Desarrollo Educativo ISSN 2007 - 7467

Tabla 6. Resultados por dimensión y ciclo escolar de estudiantes en LSC

\begin{tabular}{|c|c|c|c|c|c|c|c|c|c|c|c|c|c|c|c|c|c|c|c|c|}
\hline \multirow[t]{4}{*}{ D } & \multicolumn{8}{|c|}{2018} & \multicolumn{8}{|c|}{2019} & \multirow{2}{*}{\multicolumn{4}{|c|}{$\begin{array}{c}\text { Acumulado } \\
\text { cuatro cohortes }\end{array}$}} \\
\hline & \multicolumn{4}{|c|}{ Ene-Jun } & \multicolumn{4}{|c|}{ Ago-Dic } & \multicolumn{4}{|c|}{ Ene-Jun } & \multicolumn{4}{|c|}{ Ago-Dic } & & & & \\
\hline & \multicolumn{2}{|c|}{ AD } & \multicolumn{2}{|c|}{ IN } & \multicolumn{2}{|c|}{ AD } & \multicolumn{2}{|c|}{ IN } & \multicolumn{2}{|c|}{ AD } & \multicolumn{2}{|c|}{ IN } & \multicolumn{2}{|c|}{ AD } & \multicolumn{2}{|c|}{ IN } & \multicolumn{2}{|c|}{ AD } & \multicolumn{2}{|c|}{ IN } \\
\hline & $\begin{array}{l}\mathbf{A} \\
\mathbf{b}\end{array}$ & $\mathbf{R}$ & $\begin{array}{l}\mathbf{A} \\
\mathbf{b}\end{array}$ & $\mathbf{R}$ & $\begin{array}{l}\mathbf{A} \\
\mathbf{b}\end{array}$ & $\mathbf{R}$ & $\begin{array}{l}\mathbf{A} \\
\mathbf{b}\end{array}$ & $\mathbf{R}$ & $\begin{array}{l}\mathbf{A} \\
\mathbf{b}\end{array}$ & $\mathbf{R}$ & $\begin{array}{l}\mathbf{A} \\
\mathbf{b}\end{array}$ & $\mathbf{R}$ & $\begin{array}{l}\mathbf{A} \\
\mathbf{b}\end{array}$ & $\mathbf{R}$ & $\begin{array}{l}\mathbf{A} \\
\mathbf{b}\end{array}$ & $\mathbf{R}$ & $\mathbf{A b}$ & $\mathbf{R}$ & $\mathbf{A b}$ & $\mathbf{R}$ \\
\hline $\mathbf{A F}$ & $\begin{array}{l}1 \\
5\end{array}$ & $\begin{array}{l}5 \\
8\end{array}$ & 11 & 42 & $\begin{array}{l}4 \\
5\end{array}$ & 5 & $\begin{array}{l}4 \\
0\end{array}$ & 47 & 7 & $\begin{array}{l}3 \\
5\end{array}$ & $\begin{array}{l}1 \\
3\end{array}$ & $\begin{array}{l}6 \\
5\end{array}$ & $\begin{array}{l}5 \\
3\end{array}$ & $\begin{array}{l}5 \\
6\end{array}$ & $\begin{array}{l}4 \\
2\end{array}$ & 44 & $\begin{array}{c}12 \\
0\end{array}$ & $\begin{array}{l}5 \\
3\end{array}$ & $\begin{array}{c}10 \\
6\end{array}$ & 47 \\
\hline EF & $\begin{array}{l}1 \\
7\end{array}$ & $\begin{array}{l}6 \\
5\end{array}$ & 9 & 35 & $\begin{array}{l}3 \\
7\end{array}$ & 4 & $\begin{array}{l}4 \\
8\end{array}$ & 56 & $\begin{array}{l}1 \\
4\end{array}$ & $\begin{array}{l}7 \\
0\end{array}$ & 6 & $\begin{array}{l}3 \\
0\end{array}$ & $\begin{array}{l}4 \\
6\end{array}$ & $\begin{array}{l}4 \\
8\end{array}$ & $\begin{array}{l}4 \\
9\end{array}$ & 52 & $\begin{array}{c}11 \\
4\end{array}$ & $\begin{array}{l}5 \\
0\end{array}$ & $\begin{array}{c}11 \\
2\end{array}$ & 50 \\
\hline $\mathbf{D}$ & 9 & $\begin{array}{l}3 \\
5\end{array}$ & 17 & 65 & $\begin{array}{l}2 \\
7\end{array}$ & $\begin{array}{l}3 \\
2\end{array}$ & $\begin{array}{l}5 \\
8\end{array}$ & 68 & 7 & $\begin{array}{l}3 \\
5\end{array}$ & $\begin{array}{l}1 \\
3\end{array}$ & $\begin{array}{l}6 \\
5\end{array}$ & $\begin{array}{l}3 \\
6\end{array}$ & $\begin{array}{l}3 \\
8\end{array}$ & $\begin{array}{l}5 \\
9\end{array}$ & 62 & 79 & $\begin{array}{l}3 \\
5\end{array}$ & $\begin{array}{c}14 \\
7\end{array}$ & 65 \\
\hline $\mathbf{L}$ & 5 & $\begin{array}{l}1 \\
9\end{array}$ & 21 & 81 & 9 & 1 & $\begin{array}{l}7 \\
6\end{array}$ & 89 & 2 & $\begin{array}{l}1 \\
0\end{array}$ & $\begin{array}{l}1 \\
8\end{array}$ & $\begin{array}{l}9 \\
0\end{array}$ & $\begin{array}{l}1 \\
3\end{array}$ & $\begin{array}{l}1 \\
4\end{array}$ & $\begin{array}{l}8 \\
2\end{array}$ & 86 & 29 & $\begin{array}{l}1 \\
3\end{array}$ & $\begin{array}{c}19 \\
7\end{array}$ & 87 \\
\hline TE & $\begin{array}{l}1 \\
2\end{array}$ & $\begin{array}{l}4 \\
6\end{array}$ & 14 & 54 & $\begin{array}{l}4 \\
5\end{array}$ & $\begin{array}{l}5 \\
3\end{array}$ & $\begin{array}{l}4 \\
0\end{array}$ & 47 & $\begin{array}{l}1 \\
0\end{array}$ & $\begin{array}{l}5 \\
0\end{array}$ & $\begin{array}{l}1 \\
0\end{array}$ & $\begin{array}{l}5 \\
0\end{array}$ & $\begin{array}{l}4 \\
9\end{array}$ & $\begin{array}{l}5 \\
2\end{array}$ & $\begin{array}{l}4 \\
6\end{array}$ & 48 & $\begin{array}{c}11 \\
6\end{array}$ & $\begin{array}{l}5 \\
1\end{array}$ & $\begin{array}{c}11 \\
0\end{array}$ & 49 \\
\hline $\mathbf{P P}$ & $\begin{array}{l}1 \\
5\end{array}$ & $\begin{array}{l}5 \\
8\end{array}$ & 11 & 42 & $\begin{array}{l}4 \\
5\end{array}$ & $\begin{array}{l}5 \\
3\end{array}$ & $\begin{array}{l}4 \\
0\end{array}$ & 47 & $\begin{array}{l}1 \\
0\end{array}$ & $\begin{array}{l}5 \\
0\end{array}$ & $\begin{array}{l}1 \\
0\end{array}$ & $\begin{array}{l}5 \\
0\end{array}$ & $\begin{array}{l}4 \\
6\end{array}$ & $\begin{array}{l}4 \\
8\end{array}$ & $\begin{array}{l}4 \\
9\end{array}$ & 52 & $\begin{array}{c}11 \\
6\end{array}$ & $\begin{array}{l}5 \\
1\end{array}$ & $\begin{array}{c}11 \\
0\end{array}$ & 49 \\
\hline $\mathrm{C}$ & $\begin{array}{l}1 \\
1\end{array}$ & $\begin{array}{l}4 \\
2\end{array}$ & 15 & 58 & $\begin{array}{l}4 \\
0\end{array}$ & $\begin{array}{l}4 \\
7\end{array}$ & $\begin{array}{l}4 \\
5\end{array}$ & 53 & 9 & $\begin{array}{l}4 \\
5\end{array}$ & $\begin{array}{l}1 \\
1\end{array}$ & $\begin{array}{l}5 \\
5\end{array}$ & $\begin{array}{l}4 \\
0\end{array}$ & $\begin{array}{l}4 \\
2\end{array}$ & $\begin{array}{l}5 \\
5\end{array}$ & 58 & $\begin{array}{c}10 \\
0\end{array}$ & $\begin{array}{l}4 \\
4\end{array}$ & $\begin{array}{c}12 \\
6\end{array}$ & 56 \\
\hline $\mathbf{A}$ & $\begin{array}{l}2 \\
1\end{array}$ & $\begin{array}{l}8 \\
1\end{array}$ & 5 & 19 & $\begin{array}{l}5 \\
9\end{array}$ & $\begin{array}{l}6 \\
9\end{array}$ & $\begin{array}{l}2 \\
6\end{array}$ & 31 & $\begin{array}{l}1 \\
3\end{array}$ & $\begin{array}{l}6 \\
5\end{array}$ & 7 & $\begin{array}{l}3 \\
5\end{array}$ & $\begin{array}{l}6 \\
4\end{array}$ & $\begin{array}{l}6 \\
7\end{array}$ & $\begin{array}{l}3 \\
1\end{array}$ & 33 & $\begin{array}{c}15 \\
7\end{array}$ & $\begin{array}{l}6 \\
9\end{array}$ & 69 & 31 \\
\hline
\end{tabular}

Notas: $\mathrm{D}=$ Dimensiones; $\mathrm{AF}=\mathrm{Ambiente}$ físico $\mathrm{EF}=$ Estado fisiológico; $\mathrm{D}=$ Distribución del tiempo; L = Lectura; $\mathrm{TE}=$ Técnicas de estudio; $\mathrm{PP}=$ Preparación de pruebas; $\mathrm{C}=$ Concentración; $\mathrm{A}=$ Actitud hacia el estudio. $\mathrm{AD}=$ Hábitos adecuados; $\mathrm{IN}=$ Hábitos inadecuados; $\mathrm{Ab}=$ Valor absoluto; $\mathrm{R}=$ Valor relativo.

Fuente: Elaboración propia

En cuanto a los resultados que arroja la participación de estudiantes de nuevo ingreso que corresponden a la LIDS (en este programa solo se tienen datos de tres cohortes), estos indican que las áreas de oportunidad detectadas son similares a las de LSC, pues únicamente la dimensión Actitud hacia el estudio es ponderada con hábitos aceptables por $67 \%$ de los participantes, mientras que en las otras siete dimensiones más de la mitad de los encuestados declaran hábitos inaceptables. En términos cuantificables, las dimensiones muestran los siguientes promedios: Lectura (85\%), Distribución de tiempo (80\%), Preparación de pruebas (63\%), Concentración (59\%), Técnicas de estudio (58\%), Estado fisiológico (56\%) y Ambiente físico (51\%). 


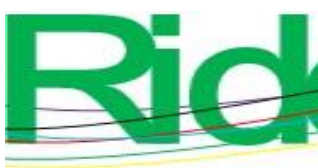

Revista Iberoamericana para la Investigación y el Desarrollo Educativo ISSN 2007 - 7467

Tabla 7. Resultados por dimensión y ciclo escolar de estudiantes de LIDS

\begin{tabular}{|c|c|c|c|c|c|c|c|c|c|c|c|c|c|c|c|c|}
\hline \multirow[t]{4}{*}{ D } & \multicolumn{8}{|c|}{2018} & \multirow{2}{*}{\multicolumn{4}{|c|}{$\begin{array}{c}2019 \\
\text { Ago-Dic }\end{array}$}} & \multirow{2}{*}{\multicolumn{4}{|c|}{$\begin{array}{c}\text { Acumulado tres } \\
\text { cohortes }\end{array}$}} \\
\hline & \multicolumn{4}{|c|}{ Ene-Jun } & \multicolumn{4}{|c|}{ Ago-Dic } & & & & & & & & \\
\hline & \multicolumn{2}{|c|}{ AD } & \multicolumn{2}{|c|}{ IN } & \multicolumn{2}{|c|}{ AD } & \multicolumn{2}{|c|}{ IN } & \multicolumn{2}{|c|}{ AD } & \multicolumn{2}{|c|}{ IN } & \multicolumn{2}{|c|}{ AD } & \multicolumn{2}{|c|}{ IN } \\
\hline & $\mathbf{A b}$ & $\mathbf{R}$ & $\mathbf{A b}$ & $\mathbf{R}$ & $\mathbf{A b}$ & $\mathbf{R}$ & $\mathbf{A b}$ & $\mathbf{R}$ & $\mathbf{A b}$ & $\mathbf{R}$ & $\mathbf{A b}$ & $\mathbf{R}$ & $\mathbf{A b}$ & $\mathbf{R}$ & $\mathbf{A b}$ & $\mathbf{R}$ \\
\hline $\mathbf{A F}$ & 9 & 53 & 8 & 47 & 17 & 53 & 15 & 47 & 26 & 45 & 32 & 55 & 52 & 49 & 55 & 51 \\
\hline EF & 11 & 65 & 6 & 35 & 15 & 47 & 17 & 53 & 21 & 36 & 37 & 64 & 47 & 44 & 60 & 56 \\
\hline DT & 5 & 29 & 12 & 71 & 7 & 22 & 25 & 78 & 9 & 16 & 49 & 84 & 21 & 20 & 86 & 80 \\
\hline $\mathbf{L}$ & 2 & 12 & 15 & 88 & 7 & 22 & 25 & 78 & 7 & 12 & 51 & 88 & 16 & 15 & 91 & 85 \\
\hline TE & 6 & 35 & 11 & 65 & 11 & 34 & 21 & 66 & 28 & 48 & 30 & 52 & 45 & 42 & 62 & 58 \\
\hline $\mathbf{P P}$ & 9 & 53 & 8 & 47 & 10 & 31 & 22 & 69 & 21 & 36 & 37 & 64 & 40 & 37 & 67 & 63 \\
\hline $\mathrm{C}$ & 8 & 47 & 9 & 53 & 16 & 50 & 16 & 50 & 20 & 34 & 38 & 66 & 44 & 41 & 63 & 59 \\
\hline $\mathbf{A E}$ & 13 & 76 & 4 & 24 & 22 & 69 & 10 & 41 & 37 & 64 & 21 & 36 & 72 & 67 & 35 & 33 \\
\hline
\end{tabular}

Notas: $\mathrm{D}=$ Dimensiones; $\mathrm{AF}=\mathrm{Ambiente}$ físico $; \mathrm{EF}=$ Estado fisiológico; $\mathrm{D}=$ Distribución

del tiempo; $\mathrm{L}=$ Lectura; $\mathrm{TE}=$ Técnicas de estudio; $\mathrm{PP}=$ Preparación de pruebas; $\mathrm{C}=$

Concentración; $\mathrm{A}=$ Actitud hacia el estudio. $\mathrm{AD}=$ Hábitos adecuados; $\mathrm{IN}=$ Hábitos inadecuados; $\mathrm{Ab}=$ Valor absoluto; $\mathrm{R}=$ Valor relativo.

Fuente: Elaboración propia

Estos resultados se precisan en la figura 1, la cual permite visualizar que aun cuando existen coincidencias en los resultados que sobre hábitos adecuados declaran los estudiantes de los programas de LC, LA y LGT, las diferencias son significativas cuando se comparan con los resultados que arroja LSC y LIDS, lo que denota la necesidad de instrumentar medidas remediales de forma diferenciada. En otras palabras, no puede hablarse de un programa genérico para mejorar hábitos de estudio para todos los estudiantes de nuevo ingreso, pues entre estos existen diferentes necesidades y carencias. Así, puede observarse que los estudiantes matriculados en la LGT son los que declaran una mejor actitud hacia el estudio, con porcentajes superiores a $80 \%$, mientras que quienes se matriculan en LC expresan mayores hábitos aceptables en Distribución del tiempo. La dimensión Estado fisiológico es la declarada con mayores hábitos aceptables por los estudiantes de LA; muy de cerca le siguen los alumnos de LC y LGT. El ambiente físico para estudiar es ponderado con mayores tasas de aceptación por estudiantes de LSC y los problemas de lectura están 

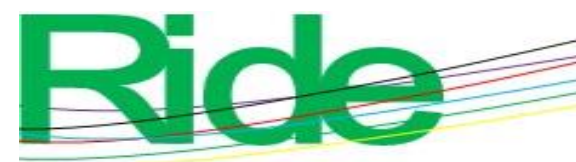

\section{Investigación y el Desarrollo Educativo \\ ISSN 2007 - 7467}

presentes en los estudiantes de los cinco programas: en ninguno se rebasan tasas aceptables más allá de $30 \%$, y en LSC y LIDS este problema se acentúa inclusive aún más.

Figura 1. Resultado promedio de las tres cohortes, por dimensión, por programa educativo

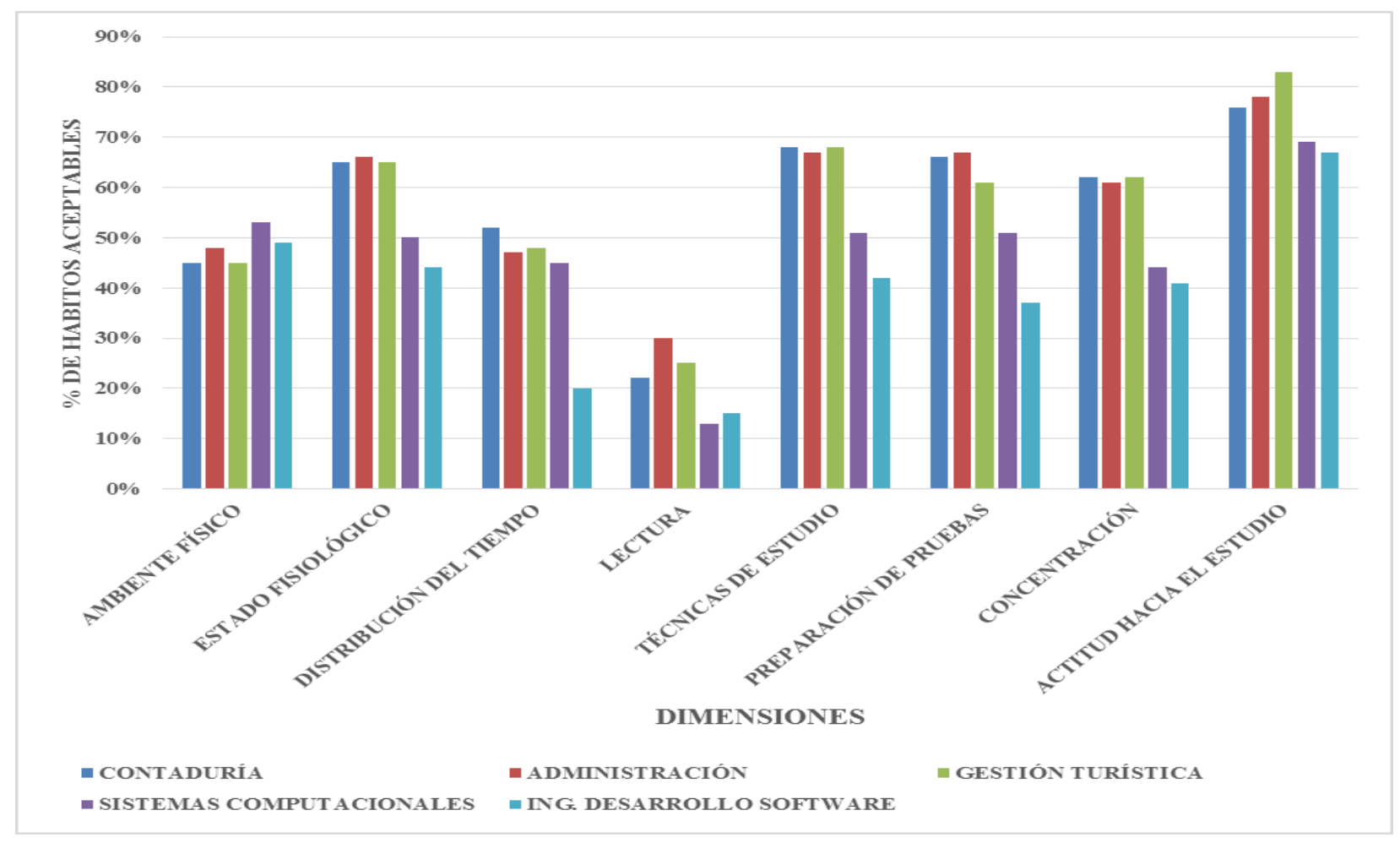

Fuente: Elaboración propia

Los datos anteriores representan los promedios obtenidos de las respuestas de los estudiantes respecto a la práctica de hábitos de estudio (adecuados e inadecuados); sin embargo, también es importante identificar el número de dimensiones del total de las ocho exploradas que cada estudiante declara como aceptable, por ello en la tabla 8 se presentan por licenciatura el número de estudiantes y el número de dimensiones que declararon aceptables, desagregado por cohortes y al final de cada programa se muestra el total acumulado.

De manera que, a nivel global, existe en promedio $23 \%$ de estudiantes de nuevo ingreso que únicamente declaran hábitos aceptables en dos o menos de las ocho dimensiones exploradas (aunque por licenciatura, este promedio debe analizarse con mayor profundidad, pues en LC la proporción es de $29 \%$, en LA de $21 \%$, en LGT es de $17 \%$, en LSC $32 \%$ y en LIDS $44 \%$ ); $26 \%$ declara hábitos aceptables entre tres y cuatro dimensiones; $30 \%$ entre 

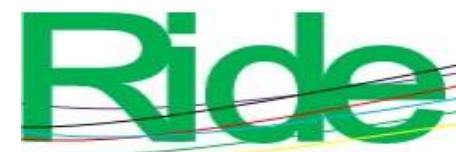

Revista Iberoamericana para la Investigación y el Desarrollo Educativo

ISSN 2007 - 7467

cinco y seis dimensiones, y solo $21 \%$ declara siete u ocho dimensiones con hábitos aceptables.

Estos resultados evidencian la necesidad de diseñar programas de intervención académica en los primeros semestres de las licenciaturas que incluyan acciones orientadas al fomento y mejora de la lectura, la práctica de técnicas adecuadas de distribución de tiempo, la mejora de la concentración y la creación de las condiciones físicas propicias para estudiar, No hay que perder de vista la consideración de que en la universidad aún se puede y debe perfeccionar o retroalimentar los procesos metacognitivos en los estudiantes, lo que seguramente demandará participación activa de docentes y autoridades, así como ignorar la premisa de que los alumnos al arribar a este nivel educativo ya poseen a cabalidad las actividades comunicativas relacionadas con escuchar, hablar, leer y escribir (Barrio del Campo, Borragá, Pérez y Castro, 2005). Para ello será necesario considerar la capacitación de los docentes en estas tareas, ya que en ocasiones suelen ser especialistas en un área de conocimiento específica, pero sin la base didáctica necesaria para realizar el quehacer docente (Vidal y Manríquez, 2016), ya que se insertaron en la docencia como opción de vida laboral presentando necesidades de actualización pedagógica (López, García, Díaz, 2018). 


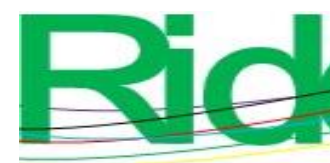

Tabla 8: Dimensiones aceptables por programa educativo

\begin{tabular}{|c|c|c|c|c|c|c|c|c|c|c|}
\hline \multirow{2}{*}{$\begin{array}{l}\text { Programa } \\
\text { educativo }\end{array}$} & \multicolumn{10}{|c|}{ Núm. de dimensiones aceptables } \\
\hline & $\mathbf{0}$ & 1 & 2 & 3 & 4 & 5 & 6 & 7 & 8 & Total \\
\hline $\begin{array}{c}\text { LC } \\
\text { E-J } 2018\end{array}$ & 3 & 8 & 4 & 18 & 15 & 39 & 9 & 15 & 12 & 123 \\
\hline A-D 2018 & 5 & 10 & 18 & 15 & 27 & 16 & 18 & 19 & 7 & 135 \\
\hline E-J 2019 & 1 & 4 & 15 & 10 & 15 & 14 & 18 & 25 & 10 & 112 \\
\hline A-D 2019 & 5 & 7 & 11 & 14 & 19 & 13 & 20 & 14 & 7 & 110 \\
\hline Total & 14 & 29 & 48 & 57 & 76 & 82 & 65 & 73 & 36 & 480 \\
\hline Relativo & $3 \%$ & $6 \%$ & $10 \%$ & $12 \%$ & $16 \%$ & $17 \%$ & $14 \%$ & $15 \%$ & $7 \%$ & $100 \%$ \\
\hline Acumulado & $3 \%$ & $9 \%$ & $29 \%$ & $31 \%$ & $47 \%$ & $64 \%$ & $78 \%$ & $93 \%$ & $100 \%$ & \\
\hline $\begin{array}{c}\text { LA } \\
\text { E-J } 2018\end{array}$ & 2 & 8 & 13 & 8 & 10 & 23 & 27 & 22 & 11 & 124 \\
\hline A-D 2018 & 2 & 5 & 12 & 11 & 17 & 22 & 23 & 17 & 10 & 119 \\
\hline E-J 2019 & 4 & 7 & 17 & 4 & 12 & 14 & 17 & 19 & 10 & 104 \\
\hline A-D2019 & 7 & 11 & 8 & 14 & 15 & 17 & 19 & 6 & 9 & 106 \\
\hline Total & 15 & 31 & 50 & 37 & 54 & 76 & 86 & 64 & 40 & 453 \\
\hline Relativo & $3 \%$ & $7 \%$ & $11 \%$ & $8 \%$ & $12 \%$ & $17 \%$ & $19 \%$ & $14 \%$ & $9 \%$ & $100 \%$ \\
\hline Acumulado & $3 \%$ & $10 \%$ & $21 \%$ & $29 \%$ & $41 \%$ & $58 \%$ & $77 \%$ & $91 \%$ & $100 \%$ & \\
\hline $\begin{array}{c}\text { LGT } \\
\text { E-J } 2018\end{array}$ & 0 & 3 & 7 & 4 & 12 & 16 & 20 & 19 & 6 & 87 \\
\hline A-D 2018 & 3 & 5 & 6 & 11 & 13 & 12 & 15 & 24 & 6 & 95 \\
\hline E-J 2019 & 4 & 3 & 6 & 13 & 12 & 11 & 11 & 18 & 2 & 80 \\
\hline A-D 2019 & 7 & 7 & 10 & 18 & 16 & 18 & 12 & 10 & 4 & 102 \\
\hline Total & 14 & 18 & 29 & 46 & 53 & 57 & 58 & 71 & 18 & 364 \\
\hline Relativo & $4 \%$ & $5 \%$ & $8 \%$ & $13 \%$ & $14 \%$ & $15 \%$ & $16 \%$ & $20 \%$ & $5 \%$ & $100 \%$ \\
\hline Acumulado & $4 \%$ & $9 \%$ & $17 \%$ & $30 \%$ & $44 \%$ & $59 \%$ & $75 \%$ & $95 \%$ & $100 \%$ & \\
\hline $\begin{array}{c}\text { LSC } \\
\text { E-J } 2018\end{array}$ & 1 & 5 & 0 & 5 & 6 & 0 & 2 & 7 & 0 & 26 \\
\hline A-D 2018 & 5 & 5 & 18 & 19 & 9 & 9 & 12 & 8 & 0 & 85 \\
\hline E-J 2019 & 0 & 1 & 2 & 9 & 4 & 0 & 4 & 0 & 0 & 20 \\
\hline
\end{tabular}




\begin{tabular}{|c|c|c|c|c|c|c|c|c|c|c|}
\hline A-D 2019 & 6 & 18 & 11 & 12 & 11 & 12 & 10 & 13 & 2 & 95 \\
\hline Total & 12 & 29 & 31 & 45 & 30 & 21 & 28 & 28 & 2 & 226 \\
\hline Relativo & $5 \%$ & $13 \%$ & $14 \%$ & $20 \%$ & $14 \%$ & $9 \%$ & $12 \%$ & $12 \%$ & $1 \%$ & $100 \%$ \\
\hline Acumulado & $5 \%$ & $18 \%$ & $32 \%$ & $52 \%$ & $66 \%$ & $75 \%$ & $87 \%$ & $99 \%$ & $100 \%$ & \\
\hline $\begin{array}{c}\text { LIDS } \\
\text { E-J 2018 }\end{array}$ & 1 & 2 & 3 & 2 & 2 & 3 & 2 & 2 & 0 & 17 \\
\hline A-D 2018 & 2 & 5 & 4 & 7 & 7 & 3 & 1 & 2 & 1 & 32 \\
\hline A-D 2019 & 5 & 12 & 13 & 5 & 8 & 9 & 3 & 3 & 0 & 58 \\
\hline Total & 8 & 19 & 20 & 14 & 17 & 15 & 6 & 7 & 1 & 107 \\
\hline $\begin{array}{c}\text { Relativo } \\
\text { Acumulado }\end{array}$ & $7 \%$ & $18 \%$ & $19 \%$ & $13 \%$ & $16 \%$ & $14 \%$ & $6 \%$ & $6 \%$ & $1 \%$ & $100 \%$ \\
\hline $\begin{array}{c}\text { Total por } \\
\text { facultad }\end{array}$ & 63 & 126 & 178 & 199 & 230 & 251 & 243 & 243 & 97 & 1630 \\
\hline Relativo & $4 \%$ & $8 \%$ & $11 \%$ & $12 \%$ & $14 \%$ & $15 \%$ & $15 \%$ & $15 \%$ & $6 \%$ & $100 \%$ \\
\hline Acumulado & $4 \%$ & $12 \%$ & $23 \%$ & $35 \%$ & $49 \%$ & $64 \%$ & $79 \%$ & $94 \%$ & $100 \%$ & \\
\hline
\end{tabular}

Fuente: Elaboración propia

\section{Discusión}

Abordar los hábitos de estudio que declaran practicar quienes ingresan como nuevos estudiantes en la universidad resulta fundamental como medida diagnóstica para identificar áreas de oportunidad que deben atenderse con estrategias adecuadas, y así eventualmente disminuir índices de rezago académico e incluso de deserción escolar.

Aun cuando todos los estudiantes participantes en el estudio declaran tener una actitud favorable hacia el estudio, se ha demostrado que entre los universitarios matriculados en los cinco programas educativos analizados existen algunas diferencias destacables, específicamente en las áreas de oportunidad, pues mientras que los de LC, LA y LGT presentan tres áreas de oportunidad prioritarias: lectura, distribución del tiempo y ambiente físico (incluso con porcentajes más o menos similares); en las licenciaturas relacionadas con ciencias de la computación, las áreas de oportunidad se incrementan (siete en ambas licenciaturas). Y cuando se analizan los resultados por estudiante, se encuentra que casi la mitad (49\%) de los estudiantes encuestados declara la práctica de hábitos aceptables 


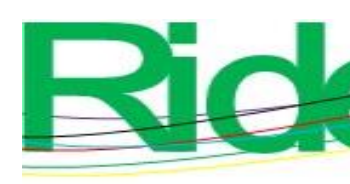

Revista Iberoamericana para la Investigación y el Desarrollo Educativo

ISSN $2007-7467$

únicamente en cuatro de las ocho dimensiones exploradas, es decir, presentan áreas de oportunidad en la mitad de las dimensiones analizadas.

Resulta urgente atender los hábitos relacionados con lectura y distribución del tiempo, área de oportunidad manifestada por los estudiantes de las cinco licenciaturas, ya que en promedio cerca de 8 (7.9) de cada 10 estudiantes tiene dificultades para realizar actividades relacionadas con la lectura y 6 (5.9) de cada 10 alumnos declara problemas para distribuir su tiempo, independientemente de las otras carencias detectadas de manera específica en cada licenciatura.

De manera general, estos hallazgos evidencian la necesidad de atender los retos que plantea la práctica de hábitos de estudio por los estudiantes de nuevo ingreso, los cuales, sin desatender los matices que se presentan, coinciden con los resultados encontrados en estudios similares. Esto es: se coincide con los 21 trabajos sistematizados por Vidal y Manríquez (2016), en donde el nivel de comprensión lectora de los universitarios es muy bajo, bajo o regular; de igual forma, con los resultados reportados por Arán y Ortega (2012), cuyos principales hallazgos refieren hábitos negativos en lo que se refiere a lectura y concentración lectora; asimismo, se conviene con lo reportado por Hernández et al. (2012) respecto a los problemas de organización y planeación del estudio — en este estudio, estos indicadores se relacionan con la distribución del tiempo-.

Aunado a lo anterior, tratándose de licenciaturas de las áreas administrativas, lo aquí descrito se ajusta a los resultados de Sarabia y Garizurieta (2006), de manera específica en las deficiencias relacionadas con distribución y administración del tiempo y técnicas de lectura, aquí englobadas en la dimensiones de Lectura y Distribución del tiempo; eso sí, no se coincide con las deficiencias detectadas en la UV respecto a la capacidad de concentración y métodos de estudio, ya que lo aquí descrito muestra como hábitos ponderados aceptablemente las variables Técnicas de estudio y Concentración.

Mientras que en comparación con el estudio de Ireta et al. (2008), se coincide como área problemática en lo que ellos denominan control y distribución del tiempo, no así en los hallazgos de técnicas de estudio y estrategias de examen, que en este estudio equivale a lo englobado en la dimensión Preparación de pruebas.

Este estudio descriptivo presenta como fortaleza principal la posibilidad de analizar datos acumulados de cuatro cohortes, lo que permite mayor consistencia en los resultados generados y estos ofrecen suficientes referentes que pueden servir como punto de partida 


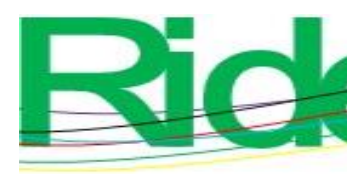

Revista Iberoamericana para la Investigación y el Desarrollo Educativo

ISSN $2007-7467$

para las acciones de mejora que deben plantearse con el propósito de facilitar el tránsito de los estudiantes durante su estancia en la universidad.

A pesar de lo anterior, debe reconocerse que existen limitantes en los resultados, debido a que lo aquí mencionado de ninguna manera puede generalizarse hacia estudiantes de las otras licenciaturas que ofrece la universidad y mucho menos hacia los estudiantes de nuevo ingreso matriculados en otras instituciones de educación superior, pues refieren en exclusiva al contexto en que se desarrollan los procesos de incorporación de nuevos estudiantes de la FCA, CI. Por otro lado, tampoco se indaga sobre las formas en que se trabaja en el aula respecto a estrategias de enseñanza que favorezcan el desarrollo del pensamiento crítico, el estudio independiente y la solución de problemas, y no se profundiza en los estilos de aprendizaje de cada estudiante.

\section{Conclusiones}

Identificar las áreas de oportunidad que presenta la práctica de hábitos de estudio en alumnos de nuevo ingreso en la universidad representa un aspecto medular en la mejora continua para optimizar los procesos de aprendizaje de los estudiantes, cuyos resultados deben considerarse como referente en los trabajos de rediseño y reestructuración curricular, en la planeación académica y representar una constante en las actividades que el docente desarrolla en el aula. De tal forma que las prácticas declaradas en los documentos institucionales se acerquen a las prácticas de uso. Puesto que desarrollar hábitos y estrategias eficaces para el estudio de los universitarios no solo permitirá concluir exitosamente la licenciatura recién iniciada, sino que sentará las bases para un desempeño eficaz en el campo profesional. No se trata solo de enseñar técnicas, métodos y procedimientos para el estudio eficaz, sino que estas sean apropiadas por los estudiantes, constituyan su práctica cotidiana y obtengan las competencias para identificar los momentos apropiados en que deban utilizarlas. Es decir, debe fomentarse en los estudiantes la autonomía del aprendizaje y la facultad para tomar decisiones, regulando el propio aprendizaje, y, por extensión, la consecución de metas, lo que implica no solo la ejecución de procedimientos estandarizados, sino el cuándo y por qué han de emplearse.

En este sentido, no debe darse por sentado que quienes ingresan a la universidad ya poseen las competencias de comunicación básica y, por lo tanto, se comunican tanto oralmente como por escrito de forma efectiva, y que han desarrollado la capacidad para 


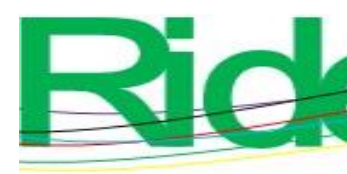

\section{Revista Iberoamericana para la Investigación y el Desarrollo Educativo ISSN 2007 - 7467}

realizar la práctica de la lectura de manera eficiente; por el contrario, hay que considerar que se trata de una tarea que la universidad no debe evadir. Para ello, no solo se plantea la necesidad de fomentar hábitos de estudio eficaces, sino de ofrecer a los discentes el apoyo efectivo para la formación integral, es decir, no se trata únicamente de que los jóvenes ingresen a la educación superior, sino apoyar el tránsito de estos durante todos los ciclos escolares que contemplan los planes de estudio, con relativas probabilidades de éxito.

De acuerdo con el test aplicado, los resultados globales muestran que en promedio cerca de la mitad $(49 \%)$ de los estudiantes de nuevo ingreso no domina más de cuatro dimensiones y que de ellos $23 \%$ no domina más de dos dimensiones; en contraste, solo $6 \%$ de los participantes declaró hábitos aceptables en las ocho dimensiones analizadas. Destacan como áreas de oportunidad principales la lectura, la distribución del tiempo, el ambiente físico y en algunos casos la concentración y la preparación de pruebas.

Los hallazgos descritos presentan similitud con los encontrados por estudios análogos: la lectura es el reto a atender de manera preponderante con los estudiantes de nuevo ingreso, sin olvidar las otras importantes áreas de oportunidad que en este estudio se identifican. Los resultados descritos permiten sugerir que los programas de atención que se instrumenten deben ser diferenciados, pues, como se ha visto, aun cuando existen deficiencias genéricas (en las dimensiones de Lectura y Distribución del tiempo, por citar las principales), no todos los estudiantes presentan los mismos problemas; tampoco tienen los mismos niveles de deficiencias y en cada licenciatura existen matices que son importantes considerar.

Por último, vale la pena destacar que los resultados aquí reportados se basan en los hábitos declarados por los estudiantes encuestados, de tal forma que, basándose en el llamado conocimiento declarativo, los estudiantes participantes dicen lo que hacen, no lo que en realidad practican o realizan cuando de actividades de estudio se trata.

Una posible vertiente a desarrollar en estudios posteriores será vincular estos resultados con la información que sobre rendimiento académico acumulen estos estudiantes durante su estancia universitaria, así como con aspectos socioculturales, familiares y académicos de los padres. Asimismo, a partir de estos resultados podrá indagarse en estudios posteriores las competencias docentes que requiere la atención de la problemática aquí descrita y la situación que prevalece en la planta académica de la facultad respecto a las necesidades formativas para la atención adecuada y oportuna en las aulas. 


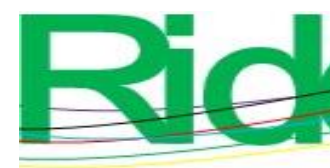

Revista Iberoamericana para la Investigación y el Desarrollo Educativo ISSN $2007-7467$

\section{Referencias}

Abarca, M. S., Gómez, M. T. y Covarrubias, M. de L. (2015). Análisis de los factores que contribuyen al éxito académico en estudiantes universitarios: estudio de cuatro casos de la Universidad de Colima. Revista Internacional de Educación y Aprendizaje, 3(2). Recuperado de https://doi.org/10.37467/gka-revedu.v3.593.

Andrade, I., Facio, S., Quiroz, A., Alemán, L., Flores, M. y Rosales, M. (2018). Actitud, hábitos de estudio y rendimiento académico: Abordaje desde la teoría de la acción razonada. Revista Enfermería Universitaria, 15(4), 342-351. Recuperado de https://dx.doi.org/10.22201/ eneo.23958421e.2018.4.533.

Arán, M. y Ortega, M. (2012). Enfoques de aprendizaje y hábitos de estudio en estudiantes universitarios de primer año de tres carreras de la Universidad Mayor Temuco, Chile 2011. Revista Educativa Hekademos, (11), 37-46. Recuperado de http://www.hekademos.com/hekadem os/ media/artículos/11/04.pdf.

Asociación Nacional de Universidades e Instituciones de Educación Superior [Anuies]. (2003). Programas institucionales de tutoría. Una propuesta de la Anuies para su organización y funcionamiento en las instituciones de educación superior (2. ${ }^{\mathrm{a}} \mathrm{ed}$.). México: Asociación Nacional de Universidades e Instituciones de Educación Superior.

Backhoff, E., Velasco, V. y Peón, M. (2013). Evaluación de la competencia de expresión escrita argumentativa de estudiantes universitarios. Revista de la Educación Superior, 42(167), 9-39. Recuperado de https://www.redalyc.org/pdf/604/60429658001.pdf.

Bajwa, N., Gujjar, A., Shaheen, G. y Ramzan, M. (2011). A comparative study of the study habits of the students from formal and non-formal systems of education in Pakistan. International Journal of Business \& Social Science, 2(14).

Barrio del Campo, J. A., Borragá, A., Pérez, M. y Castro, S. (2005). Potenciación de la lectura en estudiantes universitarios. Planteamientos para un reto futuro. International Journal of Developmental and Educational Psychology, 2(1), 91-105. Recuperado de http://www.redalyc.org/articulo.oa?id=349832309006.

Bedolla, S. (2018). Programa educativo de técnicas y hábitos de estudio para lograr aprendizajes sustentables en estudiantes de nuevo ingreso al nivel superior. Revista Iberoamericana de Educación, 76(2), 73-94. Recuperado de https://rieoei.org/RIE/article/view/2959. 


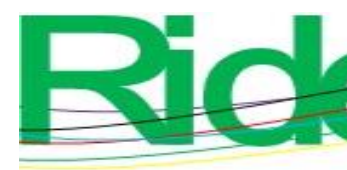

Revista Iberoamericana para la Investigación y el Desarrollo Educativo

ISSN 2007 - 7467

Bonilla, C., E. (2009). La metodología de la investigación. Práctica social y científica. En Bonilla, C., Hurtado, P. y Jaramillo, H. (coords.), La investigación. Aproximaciones a la construcción del conocimiento científico. México: Alfaomega.

Cartagena, M. (2008). Relación entre la autoeficacia, el rendimiento escolar y los hábitos de estudio en secundaria. Revista Iberoamericana sobre Calidad, Eficacia y Cambio en Educación, 6(3). Recuperado de http://www.rinace.net/arts/ vol6num3/art3.pdf.

Castañeda, S. y Ortega, I. (2004). Evaluación de estrategias de aprendizaje y orientación motivacional al estudio. En Castañeda, S. (ed.), Educación, aprendizaje y cognición. Teoría y práctica (pp. 277-299). Ciudad de México, México: Manual Moderno.

Castillo, S. y Polanco, L. (2008). Enseñar a estudiar... aprender a aprender. Didáctica del estudio. Madrid, España: Pearson Prentice Hall.

Chain, R. y Jácome, N. (2007). Perfil de ingreso y trayectoria escolar en la Universidad. Veracruz, México: Instituto de Investigaciones en Educación-Universidad Veracruzana.

Díaz, F. (2011). Metodología de diseño curricular para educación superior. México: Trillas.

Díaz, F. y Hernández, G. (2010). Estrategias docentes para un aprendizaje significativo. Una Interpretación constructivista (3. a ed.). México: McGraw-Hill.

Durán, D. (Coord.). (2011). Leemos en pareja. Tutoría entre iguales para la competencia lectora. Barcelona, España: Horsori

Elizalde, A. (2017). Hábitos de estudio. Atlante. Cuadernos de Educación y Desarrollo. Recuperado de http://www.eumed.net/rev/atlante/ 2017/08/habitos-estudio.html.

Huidobro, C. G., Gutiérrez, M. C. y Condemarín, E. (2000). A estudiar también se aprende. Metodología de estudio sesión por sesión (4. a ed.). México: Alfa Omega.

García, F., Fonseca, G. y Concha, L. (2015). Aprendizaje y rendimiento académico en educación superior: un estudio comparado. Revista Actualidades Investigativas en Educación, 15(3), 1-26. Recuperado de https://www.redalyc.org/pdf/447/44741347019.pdf.

Garrido, F. (2014). Leer y escribir para ingresar a la Educación Superior. Revista de la Educación Superior, (172), 145-150. Recuperado de http://publicaciones.anuies.mx/pdfs/revista/ Revista172_S4A1ES.pdf.

González, R. (coord.) (2014). Habilidades lingüísticas de los estudiantes de primer ingreso a las instituciones de educación superior. Área Metropolitana de la Ciudad de 


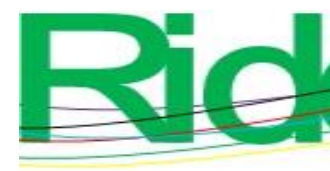

\section{Revista Iberoamericana para la Investigación y el Desarrollo Educativo ISSN 2007 - 7467}

México. México: Asociación Nacional de Universidades e Instituciones de Educación Superior.

Hernández, C., Rodríguez, N. y Vargas, Á. (2012). Los hábitos de estudio y motivación para el aprendizaje de los alumnos en tres carreras de ingeniería. Revista de la Educación Superior, 41(163), 67-87. Recuperado de http://www.redalyc.org/articulo.oa?id=6 0425380005 .

Hernández, R., Fernández, C. y Baptista L. (2014). Metodología de la investigación (6. ${ }^{\text {a ed.). }}$ México: McGraw-Hill.

Ibarrola, M. (2012). Los grandes problemas del sistema educativo mexicano. Perfiles Educativos, 34(número especial), 16-28. Recuperado de http://www.scielo.org.mx/pdf/peredu/v34 nspe/v34nspea3.pdf.

Instituto Nacional para la Evaluación de Educación [INEE]. (2011). ¿Qué saben los estudiantes al término de la educación media superior? En La educación media superior en México (pp. 117-137). México: Instituto Nacional para la Evaluación de la Educación. Recuperado de http://www.inee.edu.mx/images/informe2011/informe2011 final.pdf.

Ireta, H., González, L. O. y Pérez, M. (2008). Prácticas de estudio-aprendizaje y actitudes de estudiantes universitarios. Ponencia presentada en el XI Congreso Internacional sobre Innovaciones en Docencia e Investigación en Ciencias Económico Administrativas. Guanajuato, 2008.

Lara, M., E. (2015). Fundamentos de investigación. Un enfoque por competencias (2. ${ }^{\mathrm{a}}$ ed.). México: Alfaomega.

Lerma, V., Garrido, P. y Hernández, H. (2008). Habilidades y métodos de estudio: competencia vigente. Ponencia presentada en el XI Congreso Internacional sobre Innovaciones en Docencia e Investigación en Ciencias Económico Administrativas. Guanajuato, 2008.

Lind, A., Marchal, G., Wathen, A. (2012). Estadística aplicada a los negocios y economía (15. ${ }^{a}$ ed.). México: McGraw-Hill.

López, A., García, M. y Díaz, A. (2018). Hábitos de estudio y fracaso escolar en educación media superior. Revista Praxis Educativa ReDIE, 10(19). Recuperado de http://praxisinvestigativa.mx/assets/19_6_habi tos.pdf. 


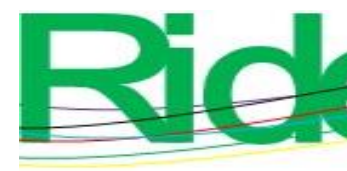

Revista Iberoamericana para la
Investigación y el Desarrollo Educativo
ISSN $2007-7467$

Malander, N. (2014). Estrategias de aprendizaje y hábitos de estudio en el nivel superior. Diferencias según el año de cursado. Apuntes Universitarios, 4(1), 9-22. Recuperado de https://dialnet.unirioja.es/servlet/articulo?codigo=4757914.

Monereo, C. (2008). La enseñanza estratégica: enseñanza para la autonomía. En Monereo, C. (coord.), Ser estratégico y autónomo aprendiendo (3. $\left.{ }^{\mathrm{a}} \mathrm{ed}.\right)$ (pp. 10-25). Barcelona, España: Grao.

Narro, R., J., Martuscelli, Q. J. y Barzana, G. E. (coords.). (2012). Plan de diez años para desarrollar el Sistema Educativo Nacional. México: Dirección General de Publicaciones y Fomento Editorial, UNAM. Recuperado de http://www.plan educativonacional.unam.mx.

Organización para la Cooperación y Desarrollo Económico [OCDE]. (2017). Panorama de la educación 2017. Nota País. Recuperado de https://www.oecd.org/education/skillsbeyond-school/EAG2017CN-Mexico-Spanish.pdf.

Pérez, M., Valenzuela, M., Díaz, A., González, J. y Núñez, J. C. (2013). Dificultades de aprendizaje en estudiantes universitarios de primer año. Atenea, (508), 135-150. Recuperado de https://scielo.conicyt.cl/scielo.php?script=sci_arttext\&pid=S071804622013000200010.

Román, M. (2013). Factores asociados al abandono y la deserción escolar en América Latina: una mirada en conjunto. Revista Iberoamericana sobre Calidad, Eficacia y Cambio en Educación, 11(2), 33-59. Recuperado de https://www.redalyc.org/pdf/551/55127024002.pdf.

Román, J., Gordillo, A. y Franco, R. (2017). Egresados de licenciatura y hábitos de estudio: Evidencia de una Institución de Educación Superior. Revista Global de Negocios, 5(3), 33-44.

Román, J., Sotelo, H. y Aguilar, D. (2016). Hábitos de estudio en egresados de Administración de la Universidad Autónoma de Chiapas. Revista Global de Negocios, 4(5), 15-26

Rowntree, D. (2001). Aprende a estudiar. Introducción programada a unas mejores técnicas de estudio. Barcelona, España: Editorial Herder.

Sarabia, M. y Garizurieta, M. (2006). Segunda etapa de la investigación sobre hábitos de estudio en estudiantes de la Facultad de Contaduría y Administración. Ponencia 


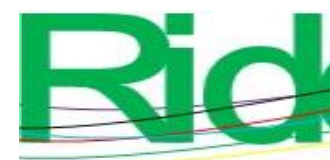

\section{Revista Iberoamericana para la Investigación y el Desarrollo Educativo \\ ISSN $2007-7467$}

presentada en el IX Congreso Internacional sobre Innovaciones en Docencia e Investigación en Ciencias Económico Administrativas. Tepic, 2006.

Secretaría de Educación Pública [SEP]. (2014). Manual para impulsar mejores hábitos de estudio en planteles de educación media superior. México: Secretaría de Educación Pública. Recuperado de https://www.gob.mx/cms /uploads/attachment/file/14844/yna_manual_3.pdf.

Universidad Autónoma de Chiapas [Unach]. (2015). Plan de estudios de la Licenciatura en Administración. Basado en competencias. México: Universidad Autónoma de Chiapas.

Universidad Autónoma de Chiapas [Unach]. (2016). Plan de estudios de la Licenciatura en Contaduría. Basado en competencias. México: Universidad Autónoma de Chiapas .

Urízar, A. (2012). Hábitos de estudio de los estudiantes del primer ciclo de la Facultad de Medicina Veterinaria y Zootecnia durante el año 2012. (tesis de licenciatura). Universidad de San Carlos de Guatemala. Recuperado de http://www.repositorio.usac.edu.gt/10696/.

Vidal, D. y Manríquez, L. (2016). El docente como mediador de la comprensión lectora en universitarios. Revista de la Educación Superior, 15(177), 95-118. Recuperado de http://publicaciones.anuies.mx/revista/177/3/4/es/el-docente-como-mediador-de-lacomprension-lectora-en-universitarios. 


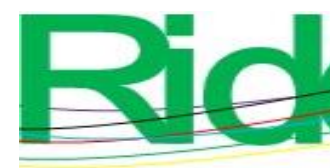

Revista Iberoamericana para la Investigación y el Desarrollo Educativo

ISSN 2007 - 7467

\begin{tabular}{|c|c|}
\hline Rol de Contribución & Autor (es) \\
\hline Conceptualización & $\begin{array}{l}\text { Juan Carlos Román Fuentes (principal)/Rafael Timoteo Franco } \\
\text { Gurría (igual) }\end{array}$ \\
\hline Metodología & $\begin{array}{l}\text { Juan Carlos Román Fuentes (principal) /Rebeca Román Julián } \\
\text { (igual) }\end{array}$ \\
\hline Software & $\begin{array}{l}\text { Juan Carlos Román Fuentes (principal) /Rafael Timoteo Franco } \\
\text { Gurría (apoyo) /Rebeca Román Julián (Apoyo) }\end{array}$ \\
\hline Validación & $\begin{array}{l}\text { Juan Carlos Román Fuentes (principal) /Rafael Timoteo Franco } \\
\text { Gurría (Apoyo) /Rebeca Román Julián (Apoyo) }\end{array}$ \\
\hline Análisis Formal & $\begin{array}{l}\text { Juan Carlos Román Fuentes (principal) /Rebeca Román Julián } \\
\text { (apoyo) }\end{array}$ \\
\hline Investigación & $\begin{array}{l}\text { Juan Carlos Román Fuentes (principal) /Rafael Timoteo Franco } \\
\text { Gurría (igual) /Rebeca Román Julián (igual) }\end{array}$ \\
\hline Recursos & $\begin{array}{l}\text { Juan Carlos Román Fuentes (principal) /Rafael Timoteo Franco } \\
\text { Gurría (apoyo) /Rebeca Román Julián (apoyo) }\end{array}$ \\
\hline Curación de datos & $\begin{array}{l}\text { Juan Carlos Román Fuentes (principal) /Rafael Timoteo Franco } \\
\text { Gurría (apoyo) }\end{array}$ \\
\hline $\begin{array}{l}\text { Escritura - Preparación del } \\
\text { borrador original }\end{array}$ & Juan Carlos Román Fuentes \\
\hline $\begin{array}{l}\text { Escritura - Revisión y } \\
\text { edición }\end{array}$ & $\begin{array}{l}\text { Juan Carlos Román Fuentes (principal) /Rafael Timoteo Franco } \\
\text { Gurría (apoyo) /Rebeca Román Julián (apoyo) }\end{array}$ \\
\hline Visualización & $\begin{array}{l}\text { Juan Carlos Román Fuentes (principal) /Rafael Timoteo Franco } \\
\text { Gurría (apoyo) /Rebeca Román Julián (apoyo) }\end{array}$ \\
\hline Supervisión & Juan Carlos Román Fuentes \\
\hline $\begin{array}{l}\text { Administración de } \\
\text { Proyectos }\end{array}$ & Juan Carlos Román Fuentes \\
\hline Adquisición de fondos & $\begin{array}{l}\text { Juan Carlos Román Fuentes (principal) /Rafael Timoteo Franco } \\
\text { Gurría (igual)/Rebeca Román Julián (igual) }\end{array}$ \\
\hline
\end{tabular}

